\title{
Effect of Vortex Generators on Corner Flow Separation Caused by Shock Wave-Boundary-Layer Interaction
}

\author{
Shunsuke Koike ${ }^{1}$ \\ Japan Aerospace Exploration Agency, Chofu, Tokyo, 182-8522, Japan \\ and \\ Holger Babinsky ${ }^{2}$ \\ Cambridge University, Cambridge, England, CB2 1PZ, United Kingdom
}

\begin{abstract}
Wind tunnel experiments were conducted to investigate the effect of vortex generators on a corner flow separation caused by an interaction between a normal shock wave and the boundary layer in a Mach 1.4 flow. The shape of the vortex generators was rectangular. The vortex generators were mounted on a bottom wall of the test section. The parameters of the vortex generators were the rotation direction of the vortex, their size and their location. When the leading edge of the vortex generators turn towards the corner, the effect of the vortex generators on the corner flow separation decreased monotonically as the vortex generators size decreased. An independent separation appeared on the bottom wall in the case. When the leading edge of the vortex generators point in the opposite direction, the flow structure was changed by the size and the location of the vortex generators. We categorized the flow structures into three modes. The effect of the vortex generators and the three modes were successfully collapsed with the location parameter normalized by the second power of the scale parameter.
\end{abstract}

\section{Nomenclature}

$A_{v} \quad=$ angle of a vortex generator to the uniform flow

$D_{v l} \quad=$ distance between a side wall and the leading edge of a vortex generator

$D_{v t} \quad=$ distance between a side wall and the trailing edge of a vortex generator

$H_{v} \quad=$ height of a vortex generator

$L_{v} \quad=$ length of a vortex generator

$\mathrm{M} \quad=$ Mach number of uniform flow

$P_{0} \quad=$ Stagnation pressure

$\operatorname{Re} \quad=$ Reynolds number

$T_{0} \quad=$ Stagnation temperature

$W_{1 B}=$ Width of the streamlines at $x=-60 \mathrm{~mm}$ on a bottom wall for an evaluation $W_{2 B}$

$W_{1 S}=$ Width of the streamlines at $x=-60 \mathrm{~mm}$ on a sidewall for an evaluation $W_{2 S}$

$W_{2 B} \quad=$ Local minimum width of the streamlines starting from $W_{1 B}$ on a bottom wall

$W_{2 B_{B} \text { Base }}=$ Local minimum width of the streamlines starting from $W_{1 B}$ on a bottom wall in the base flow case

$W_{2 S}=$ Local minimum width of the streamlines starting from $W_{l S}$ on a sidewall

$W_{2 S_{-} \text {Base }}=$ Local minimum width of the streamlines starting from $W_{1 B}$ on a sidewall in the base flow case

$X_{v s} \quad=$ location of vortex generators in the streamwise direction

$x \quad=$ streamwise direction

$x_{v} \quad=$ distance from the leading edge of a vortex generator in the streamwise direction

$y=$ vertical direction to a bottom wall

$z \quad=$ spanwise direction

$z_{v} \quad=$ distance from the leading edge of a vortex generator in the spanwise direction

\footnotetext{
${ }^{1}$ Researcher, Aeronautical Technology Directorate, Member AIAA.

${ }^{2}$ Professor, Department of Engineering. Associate Fellow AIAA.
} 
$\Delta X_{v s} \quad=$ distance between a normal shock wave and the leading edge of a vortex generator

$\delta \quad=\quad$ thickness of the boundary layer at the leading edge of a vortex generator

\section{Introduction}

$\mathrm{T}$

he boundary layer growing along a streamwise corner is generally found to separate more easily than an equivalent flat plate boundary layer because the viscous retardation of two surfaces leads to significant loss of streamwise momentum in the corner region. Here we investigate the onset of corner separation in a normalshock/boundary-layer interaction with a view to determine whether it is possible to apply flow control in this region. Figure 1 shows the conceptual diagrams of the interaction between a normal shock wave and a boundary layer in a rectangular duct. The nominal configuration of the setup is two dimensional. However, the flow structure is highly three dimensional because of the corner interaction where localized separation occurs more upstream than elsewhere. Figure 2 shows the result of a typical surface oil flow visualization in such a flow, as reported by Bruce et al ${ }^{1}$. It clearly shows that the large separation area in both corner regions of the duct starts further upstream than that in the center region. Such three-dimensional corner separations can appear in the inlet of jet engines and in the junction area between an aircraft wing and a body. Generally, the separations can cause a loss of engine performance and an increase of the aircraft drag. Thus, it is highly desirable to use flow control to suppress such separations. For the transonic wing, vortex generators (VGs) are effective to reduce the shock wave separation and to suppress the shock wave oscillation ${ }^{2-4}$. However, so far there has been no successful application of VGs ahead of corner separation ${ }^{1}$. Hence, in this research, we investigate the potential of VGs to delay or prevent corner separations. The design of the VGs under investigations is similar to the type of device used successfully on transonic aircraft wings ${ }^{3,4}$.

As shown in Fig. 3, the streamwise vortex produced by a vortex generator introduces considerable spanwise variation in boundary layer thickness. The vortex produces a thinner boundary layer in the downwash region and a thickened boundary layer in the upwash region. This variation in boundary layer properties has a considerable effect on any subsequent shock wave boundary layer interaction. Figure 4 clearly shows that the shape of the oil pattern produced by shock wave separation is curved due to the variations in boundary layer thickness. Figure 4 also shows that the trajectory of the vortex is not straight because of the induced velocity of its mirror vortex. Because corner flow separations are highly three dimensional, it is thought that these effects are more important in corner flows than on flat plates or wings. Therefore, we investigated the effect of VGs on the corner flow separation by focusing on the influence of the vortex rotation direction. In addition, we investigated the influence of the size and the location of VGs to obtain guidelines for their application in practical situations.
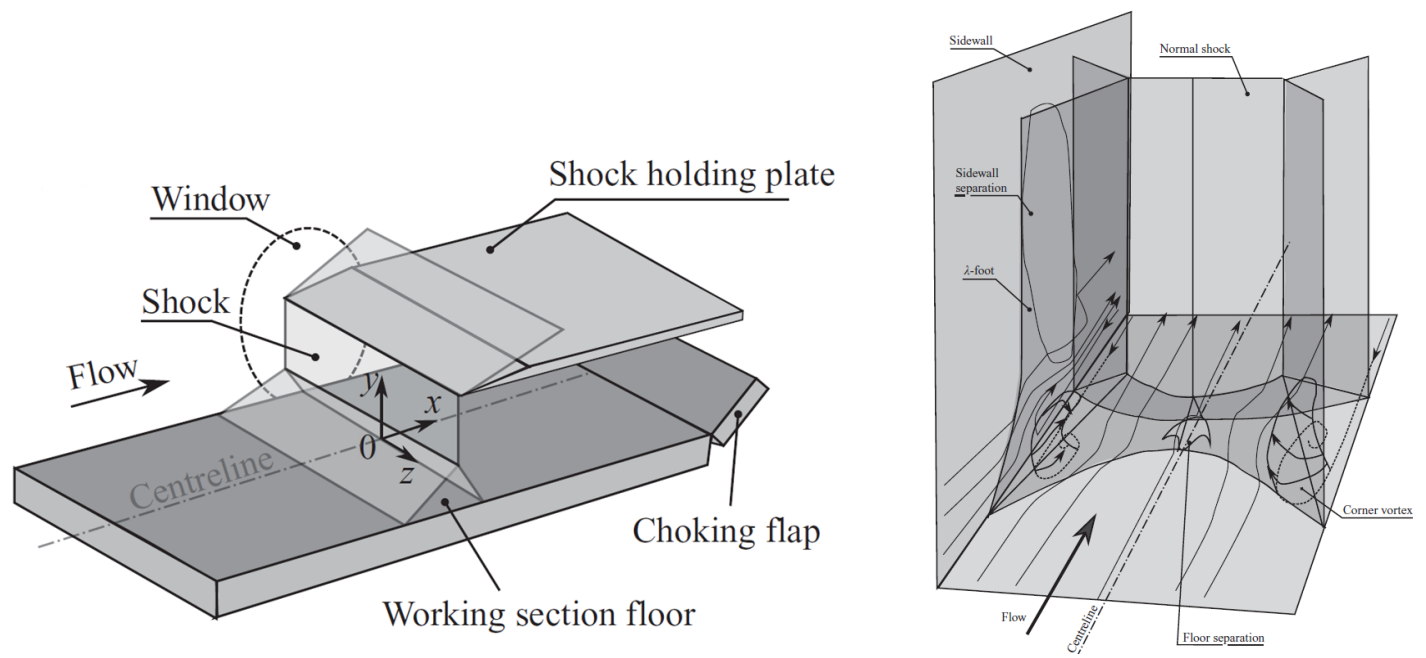

Figure 1 Conceptual figure of interaction between a normal shock wave and a boundary layer in a rectangular duct ${ }^{1}$. 


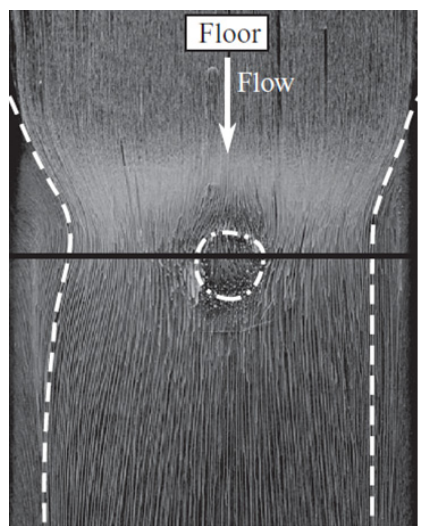

Figure 2 Oil flow pattern on the floor around the shock wave boundary layer interaction in a Mach number $=\mathbf{1 . 5}$ flow $^{1}$. Black line shows the shock wave location. White broken lines show the separation area.

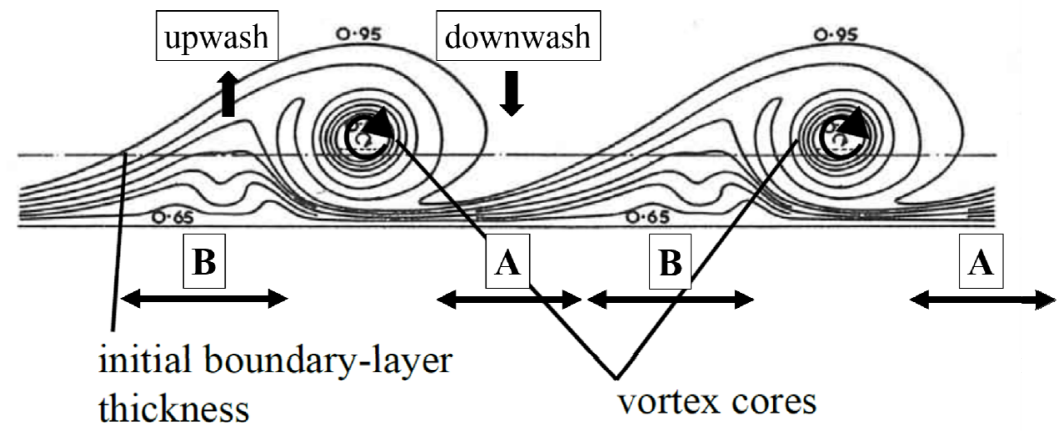

Figure 3 Boundary layer deformed by the streamwise vorte ${ }^{2}$.

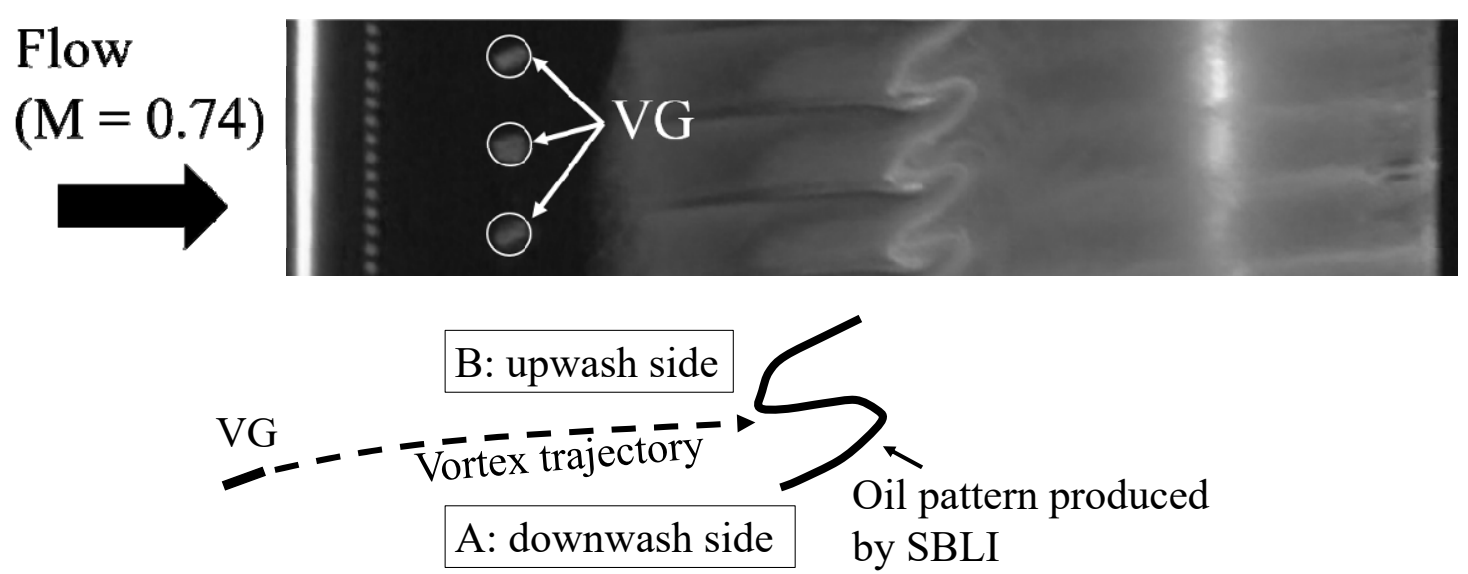

Figure 4 Oil flow pattern produced by the interaction between a shock wave and streamwise vortices on an unswept two-dimensional wing ${ }^{4}$ (Airfoil of NASA Common Research Model ${ }^{5}$ ) 


\section{Experimental method}

\section{A. Wind tunnel and shock holding system}

Experiments were performed in the Cambridge University Engineering Department supersonic wind tunnel no.1. Figure 5 shows a schematic figure of the flow field and the test section. To hold the normal shock wave at our desirable location, a shock holding plate was set in the test section ${ }^{6}$. By adjusting a chocking block mounted on the shock holding plate at the exit of the test section, the normal shock wave can be held at the test location. The distance between the shock holding plate and the bottom wall was $122 \mathrm{~mm}$. The width of the duct was $114 \mathrm{~mm}$. A right-handed coordinate system as shown in Fig. 5 is used in this paper. The flow direction, the height direction and the spanwise direction are $x, y$, and $z$, respectively. The origin of the coordinate system is on the center line of the bottom wall. The edge of the shock holding plate is the origin in the flow direction.

Table 1 summarizes the uniform flow conditions. Nominal Mach number and total pressure were set at 1.4 and $185 \mathrm{kPa}$, respectively. Total temperature was $300 \mathrm{~K} \pm 5 \mathrm{~K}$. The unit Reynolds number is estimated at 27.4 million. Typical duration time of each blow was about 20 seconds.

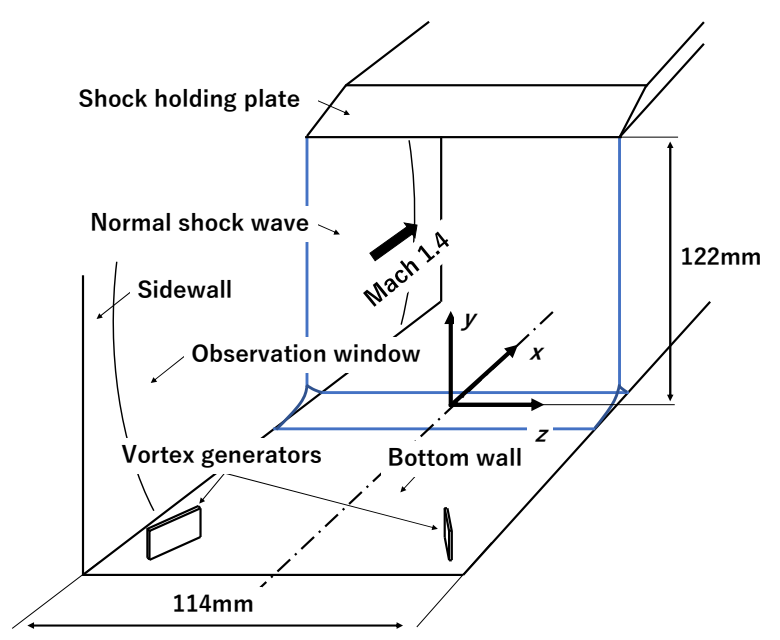

Figure 5 Schematic figure of flow field

Table 1 Nominal parameters of uniform flow

\begin{tabular}{|c|c|c|c|}
\hline $\mathrm{M}$ & unit Re $[1 / \mathrm{m}]$ & $T_{0}[\mathrm{~K}]$ & $P_{0}[\mathrm{kPa}]$ \\
\hline 1.4 & 27.4 milion & 300 & 185 \\
\hline
\end{tabular}

\section{B. Parameters of VGs}

Figure 6 shows the definition of the VG's parameters. We defined VGs whose leading edge turns towards the corner as "toe-in" VGs, and VGs whose leading edge points in the opposite direction as "toe-out" VGs. The VGs were mounted on the bottom wall in all test cases. The shape of the VG was rectangular. The main parameters were height $H_{v}$, length $L_{v}$, angle $A_{v}$, and the distance from the side wall $D_{v l}$ and $D_{v v}$. The boundary layer thickness at the leading edge of the VG $\delta$ was adopted as the representative length-scale of the boundary layer. The boundary layer thickness at this location was estimated from a straight line interpolation of the velocity profiles given in Ref. 7 .

Table 2 shows the parameters of the all test cases. In the baseline VGs tests, the VGs parameters were equivalent to these used in previous research ${ }^{3,4}$. Such VGs were found to successfully reduce transonic buffet on wings. The height of the VGs was based on the thickness of the boundary layer at $x=-100 \mathrm{~mm}$ which is the baseline case VG position. The height was $7.5 \mathrm{~mm}$ and 1.6 times the boundary layer thickness. The distances between the VGs and the sidewall, $D_{v l}$ and $D_{v t}$ are the original parameters in this research. In the baseline cases, the distances were selected to avoid any interaction between the VGs and the sidewall boundary layer. The minimum distance between the VGs and the sidewall is $7.5 \mathrm{~mm}$, which is 1.6 times the boundary layer thickness in the base VGs case. The aspect ratio of 
the VGs $L_{v} / H_{v}$ and the angle $A_{v}$ were fixed at 4 and 20 degrees, respectively. To investigate the influence of the VGs size, VGs heights of $2.5 \mathrm{~mm}$ and $5.0 \mathrm{~mm}$ were also tested. The influence of VGs location was investigated only in the toe-out VGs cases.
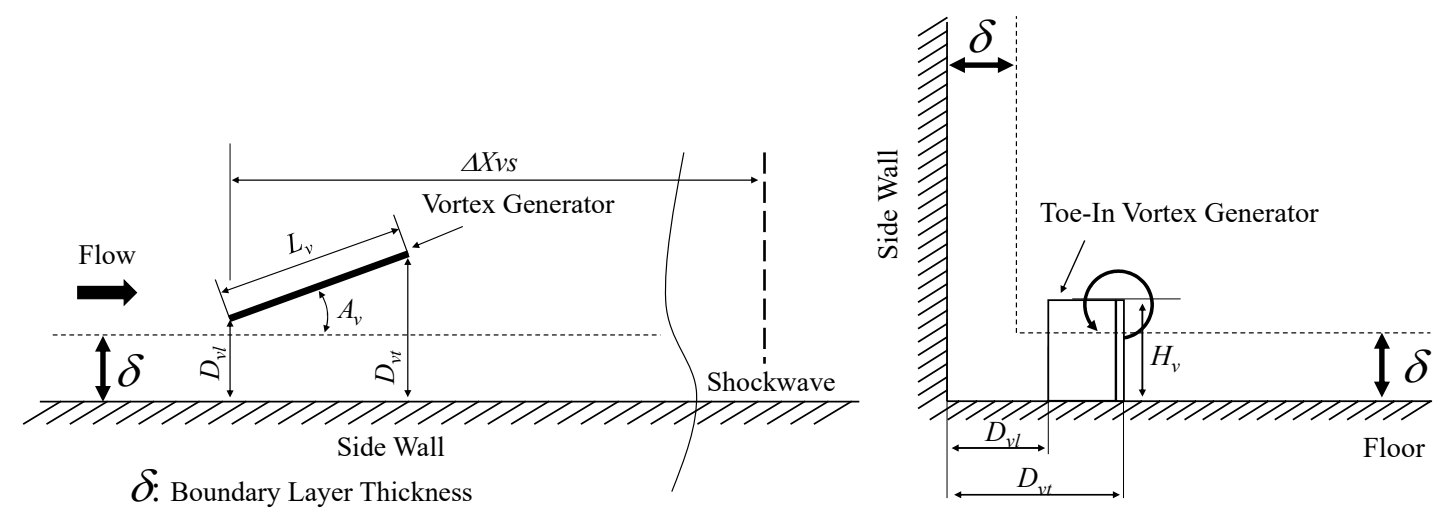

(a) Toe-in VG
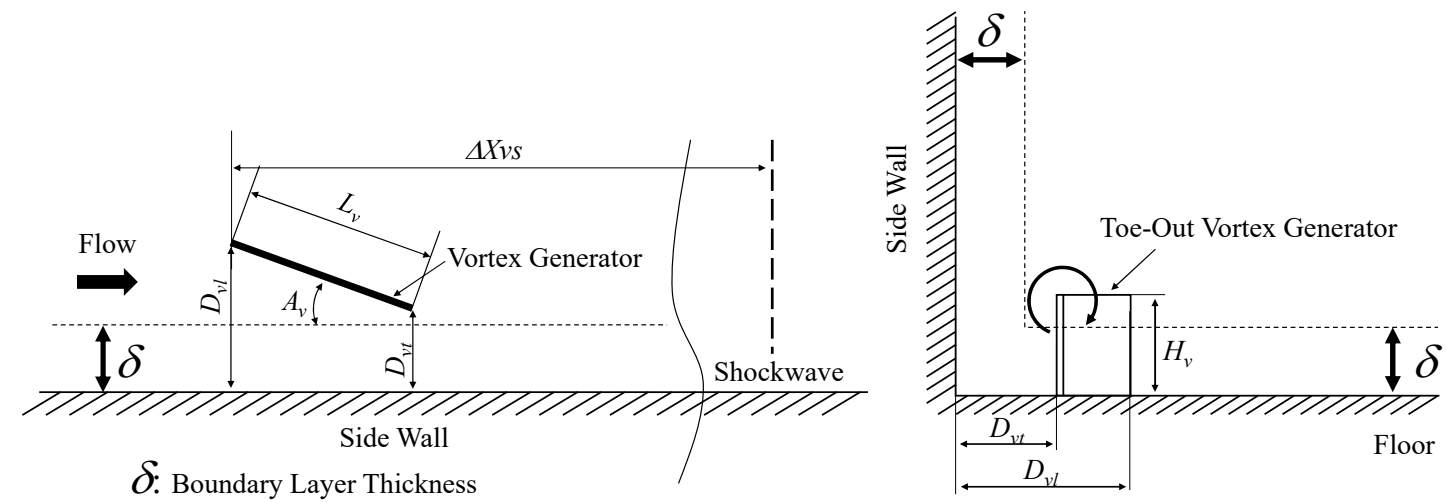

(b) Toe-out VG

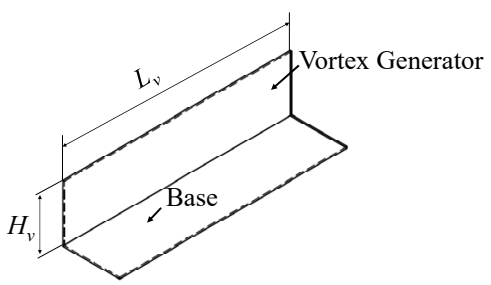

(c) Rectangular vortex generator

Figure 6 Definition of vortex generators (a)Toe-in VG, (b) Toe-out VG, (c) Shape of VG. 
Table 2 Parameters of vortex generators

Toe-out VGs

\begin{tabular}{|c|c|c|c|c|c|c|c|c|c|c|}
\hline$A_{v}$ & $H_{v}$ & $L_{v}$ & $D_{v t}$ & $\Delta X_{v s}$ & $\delta$ & $L_{v} / H_{v}$ & $H_{v} / \delta$ & $D_{v t} / \delta$ & $\Delta X_{v s} / \delta$ & \multirow{2}{*}{ Note } \\
\hline Unit [deg.] & \multicolumn{5}{|c|}{ Unit [mm] } & \multicolumn{4}{|c|}{ Dimensionless } & \\
\hline 20.0 & 7.5 & 30.0 & 7.5 & 100.0 & 4.6 & 4.0 & 1.6 & 1.6 & 21.7 & Base case \\
\hline 20.0 & 7.5 & 30.0 & 7.5 & 150.0 & 4.0 & 4.0 & 1.9 & 1.9 & 37.5 & \\
\hline 20.0 & 7.5 & 30.0 & 7.5 & 200.0 & 3.4 & 4.0 & 2.2 & 2.2 & 58.8 & \\
\hline 20.0 & 7.5 & 30.0 & 0.0 & 200.0 & 3.4 & 4.0 & 2.2 & 0.0 & 58.8 & \\
\hline 20.0 & 7.5 & 30.0 & 22.5 & 200.0 & 3.4 & 4.0 & 2.2 & 6.6 & 58.8 & \\
\hline 20.0 & 5.0 & 20.0 & 7.5 & 100.0 & 4.6 & 4.0 & 1.1 & 1.6 & 21.7 & \\
\hline 20.0 & 5.0 & 20.0 & 7.5 & 150.0 & 4.0 & 4.0 & 1.3 & 1.9 & 37.5 & \\
\hline 20.0 & 2.5 & 10.0 & 7.5 & 100.0 & 4.6 & 4.0 & 0.5 & 1.6 & 21.7 & \\
\hline 20.0 & 2.5 & 10.0 & 7.5 & 200.0 & 3.4 & 4.0 & 0.7 & 2.2 & 58.8 & \\
\hline
\end{tabular}

Toe-in VGs

\begin{tabular}{|c|c|c|c|c|c|c|c|c|c|c|}
\hline$A_{v}$ & $H_{v}$ & $L_{v}$ & $D_{v l}$ & $\Delta X_{v s}$ & $\delta$ & $L_{v} / H_{v}$ & $H_{v} / \delta$ & $D_{v l} / \delta$ & $\Delta X_{v s} / \delta$ & \multirow{2}{*}{ Note } \\
\cline { 1 - 10 } Unit [deg.] & \multicolumn{9}{|c|}{ Unit [mm] } & \multicolumn{7}{c|}{ Dimensionless } & \\
\hline 20.0 & 7.5 & 30.0 & 7.5 & 100.0 & 4.6 & 4.0 & 1.6 & 1.6 & 21.7 & Base case \\
\hline 20.0 & 5.0 & 20.0 & 7.5 & 100.0 & 4.6 & 4.0 & 1.1 & 1.6 & 21.7 & \\
\hline 20.0 & 2.5 & 10.0 & 7.5 & 100.0 & 4.6 & 4.0 & 0.5 & 1.6 & 21.7 & \\
\hline
\end{tabular}

\section{Measurement techniques}

A schlieren system is used to monitor the flow fields. The system consisted of a light source, two concave mirrors, a reflection mirror, a knife edge and a CMOS camera. A circular area of diameter $203 \mathrm{~mm}$ is visualized by the system through the observation windows. The direction of the knife edge is set in the horizontal direction to best visualize the VG's vortex. Although this is sensitive to vertical density gradients, the normal shock wave can be clearly seen because of the shadowgraph effect.

Surface oil flow is used to visualized the streamlines on the bottom wall and sidewall at $z=+57 \mathrm{~mm}$. The mixture uses a combination of Titanium Dioxide $\left(\mathrm{TiO}_{2}\right)$ powder, fluidic paraffin, and oleic acid. The ratio of the $\mathrm{TiO}_{2}$ and the fluidic paraffin is $33 \mathrm{~g}$ to $175 \mathrm{ml}$. About 10 drops of the oleic acid is added after mixing. The fluidic paraffin in the mixture evaporates during the blow. Hence, the white pattern in the pictures shows the patterns of remained $\mathrm{TiO}_{2}$ powder. The bottom wall is painted black to improve the contrast of the patterns in the pictures. The oil is painted on the bottom wall in all cases. In some tests, the oil is also painted on the sidewall. In those cases, the streamlines close to the shock holding plate can be observed on the sidewall.

A two component Laser Doppler Velocimetry (LDV) system (TSI) is used for the velocity measurement downstream of the normal shock wave. Two pairs of coherent laser beams (542nm and 532nm) are focused inside the test section. The measurement volume is $130 \mu \mathrm{m}$ in diameter. Kerosene particles with a diameter of approximately $0.5 \mu \mathrm{m}^{8}$, are used as the tracer. The measurement volume is traversed from $y=20 \mathrm{~mm}$ to $y=0 \mathrm{~mm}$ at $x=+40 \mathrm{~mm}$ and $\mathrm{z}=-47 \mathrm{~mm}$. The velocity measurements are repeated three times at the same condition. Although typical measurement accuracy was estimated to be better than $1 \mathrm{~m} / \mathrm{s}^{8}$, a larger discrepancy is locally observed between the three measurements because the particles density is locally lower than that in the typical condition. To show the discrepancy, all data is shown in the result section. It roughly shows the accuracy of the velocity data at each measurement points.

To calculate the local Mach number from the velocity data, total temperature is measured by T-type thermo couples in the settling chamber. The local Mach number at each measurement point is calculated from the total temperature and the measured velocity. A adiabatic flow is assumed in the calculation.

Pressure of the total pressure and ports on the walls is measured using a pressure scanner. The uncertainty of the pressure scanner is less than $0.1 \mathrm{kPa}$.

The pressure distributions on the bottom wall are also visualized with pressure sensitive paint (PSP). The PSP (UniCoatPSPUNC-12, ISSI) is painted directly on the metal surface of the bottom wall. The PSP is illuminated with UV light (LM2x-DM, ISSI) through the observation window. The luminescence of the PSP is recorded with a consumer CMOS camera (Nikon D7100) at a resolution of $0.04 \mathrm{pixel} / \mathrm{mm}$. After an intensity correction, a Wiener 
filter is applied to the images to reduce the influence of noise. A window size of 64 pixels $\times 64$ pixels is adopted for the procedure corresponding to $2.4 \mathrm{~mm} \times 2.4 \mathrm{~mm}$ in physical length. In-situ calibration technique with linear SternVolmer equation ${ }^{9}$ is adopted to change the intensity ratio of the images to the pressure value. Six taps on the center line of the bottom wall are used as the reference points. The root mean square of the difference between the PSP data and the pressure scanner data is roughly $5 \%$ of the measurement values.

\section{Results}

\section{A. Single VG on a flat plate}

Figure 7(a) shows the oil flow picture in the case without a shock wave. The height of the single VG was $7.5 \mathrm{~mm}$, which is the height of the base VG cases. A wavy pattern is observed on the expansion side of the VG. This indicates the footprint of the streamwise vortex. The footprint moves upward in this figure. Figure 7(b) shows the oil flow picture in the case with a normal shock wave. The location of the normal shock wave was at $x=0 \mathrm{~mm}$. A large separation appeares on the compression side of the VG because of the interaction between the normal shock wave and the boundary layer on the compression side.

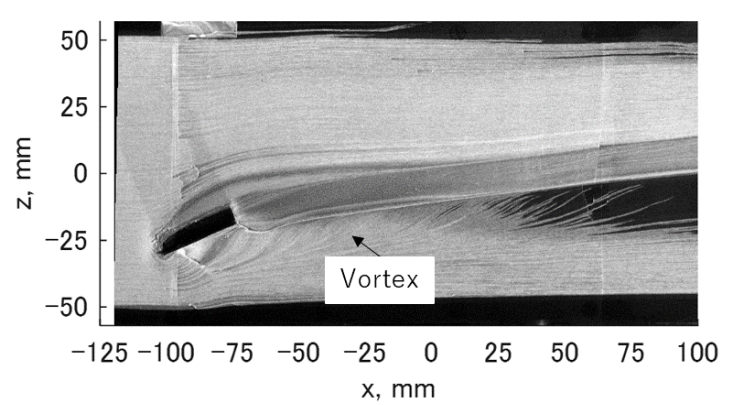

(a)No shock wave case

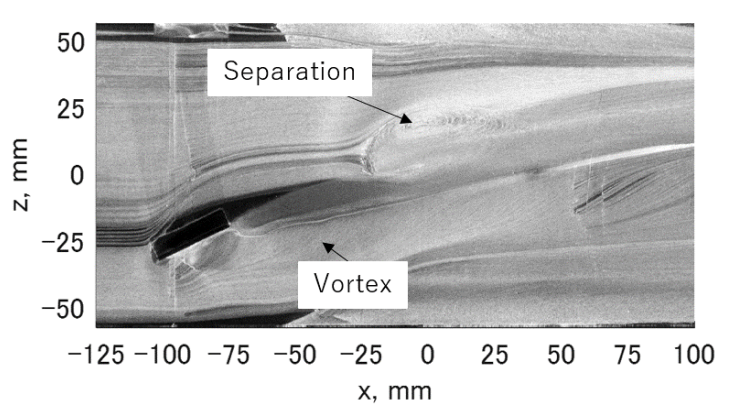

(b)Shock wave case

Figure 7 Oil flow picture of a single VG $\left(H_{v}=7.5 \mathrm{~mm}\right)$ on a flat plate.

\section{B. Influence of the VG direction}

Figure 8(a) shows a schlieren image in the case without VGs (the base flow). The location of the normal shock wave is adjusted to be at $x=0 \mathrm{~mm}$. The chocking block is then fixed based to this setting in all other cases. Because of the interaction between the normal shock wave and the boundary layer, a $\lambda$ shock wave appeares in the region close to the bottom wall. The height of the triple point is about $20 \mathrm{~mm}$.

Figure $8(\mathrm{~b})$ shows a schlieren image in the toe-in VGs case. The location of the normal shock wave is $x=10 \mathrm{~mm}$ which is further upstream from in the base flow case. The size of the $\lambda$ shock wave is larger than in the base flow case. The height of the triple point is about $40 \mathrm{~mm}$. It indicates that a larger separation appears on the bottom wall in the toe-in VGs case compared to the base flow.

Figure 8(c) shows a schlieren image of the toe-out VGs case. The location of the normal shock wave is a little upstream from in the base flow case. The size of the $\lambda$ shock wave is smaller than in the base flow case. The height of the triple point is less than $20 \mathrm{~mm}$. This indicates that the toe-out VGs reduce the size of the separation on the bottom wall.

Figure 9 shows the oil flow visualization on the sidewall and the bottom wall. The separations are observed in both corners. The streamlines on the bottom wall show attached flow in the center region.

Figure 10(a) shows oil flow pictures in the base toe-in VGs case. From the streamlines on the sidewall, it is confirmed that the VGs reduce the corner flow separation. The angle of the streamlines from $y=25 \mathrm{~mm}$ to $50 \mathrm{~mm}$ on the sidewall is lower than that in the base flow case. However, a separation appears in the center region on the bottom wall. We will refer to this as the "bottom wall separation" hereafter. The bottom wall separation in Fig. 10(a) corresponds to the separation on the compression side of the vortex in the single VG case.

Figure 10(b) shows oil flow pictures in the toe-out VGs case. From the streamlines on the bottom wall, it is confirmed that the VGs reduce the corner flow separation. The toe-out VGs lift the low momentum flow from the corner and onto the sidewall. As the result, a separation independently appears on the sidewall. We will refer to this 
as the "sidewall separation" hereafter. It seems that the sidewall separation corresponds to the separation on the compression side of the vortex in the single VG case.

Figure 11 shows the profiles of local Mach number in the vertical direction at $x=+40 \mathrm{~mm}$ and $z=-47 \mathrm{~mm}$. The values are calculated from the velocity profiles measured with LDV. The measurement location is illustrated as an open circle in Figs 9, 10 and 12. From $y=0 \mathrm{~mm}$ to $5 \mathrm{~mm}$, the local Mach numbers in the VGs cases are higher than in the base flow case. These results quantitatively confirm that the VGs successfully improve the condition of the corner flow. From $y=10 \mathrm{~mm}$ to $20 \mathrm{~mm}$, the local Mach number in the toe-out VGs case is lower than that in the base flow case. The low speed region is caused by the sidewall separation shown in Fig. 10(b).

Figure 12(a)-(c) shows the pressure distributions on the bottom wall measured with the PSP. In the toe-in VGs case, the low-pressure regions extend from the VGs to downstream of the normal shock wave. These low pressure regions are thought to be foot prints on the streamwise vortices. The distribution in the corner regions is complicated in the toe-in VGs case because of the vortex footprints. The contour lines at $x=-20 \mathrm{~mm}$ are quite straight in the toeout VGs case because the toe-out VGs reduces the corner flow separation.

The symbols and lines in Fig. 12(d) show the pressure measured by the pressure taps and PSP, respectively, on the center line of the bottom wall. The pressure increase location in the toe-in VGs case is the most upstream of all three cases. It is caused by the bottom wall separation. The pressure recovery in the toe-out VGs case is greatest on the centerline in the three cases.

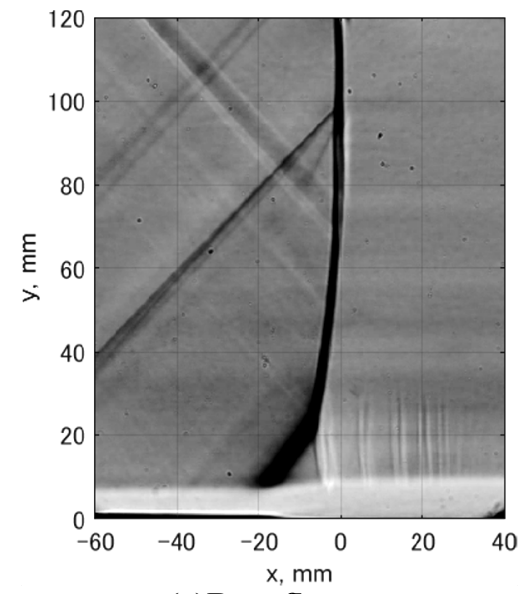

(a)Base flow

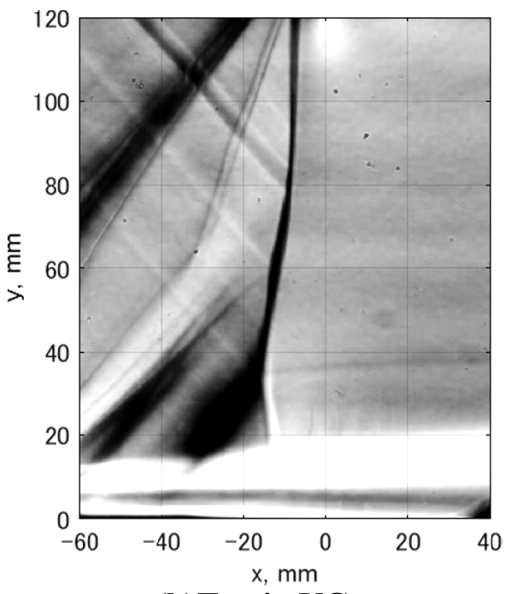

(b)Toe-in VGs

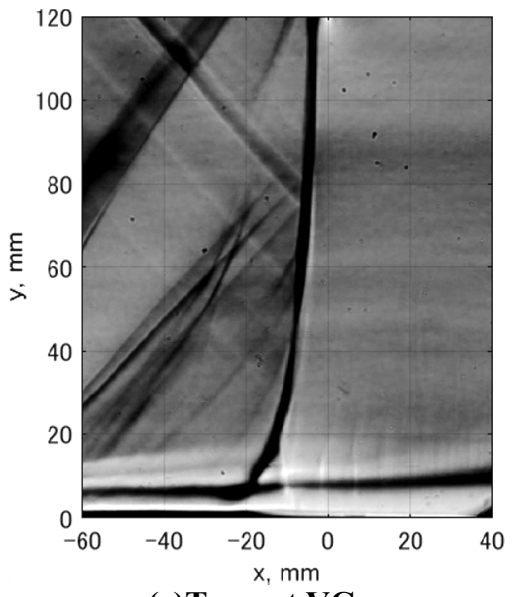

(c)Toe-out VGs

Figure 8 Schlieren images of the normal shock wave in the base flow case, the toe-in VGs case $\left(H_{v}=\right.$ $\left.7.5 \mathrm{~mm}, D_{v l}=7.5 \mathrm{~mm}, \Delta X_{v s}=100 \mathrm{~mm}\right)$ and the toe-out VGs case $\left(H_{v}=7.5 \mathrm{~mm}, D_{v t}=7.5 \mathrm{~mm}\right.$, $\left.\Delta X_{v s}=100 \mathrm{~mm}\right)$. 

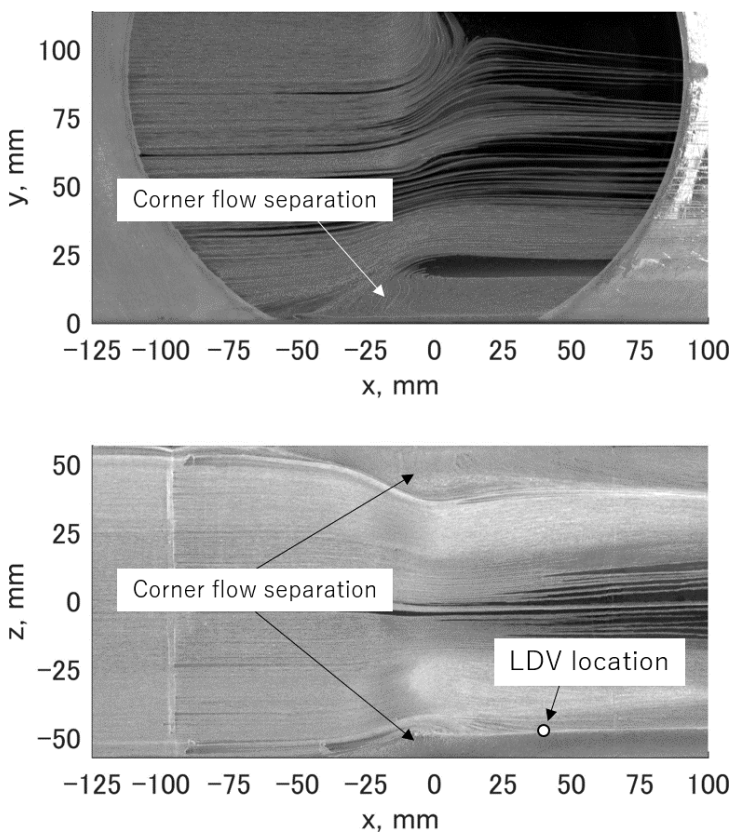

Figure 9 Oil flow pictures of the base flow.

(Top: sidewall at $z=+57 \mathrm{~mm}$, Bottom: bottom wall)
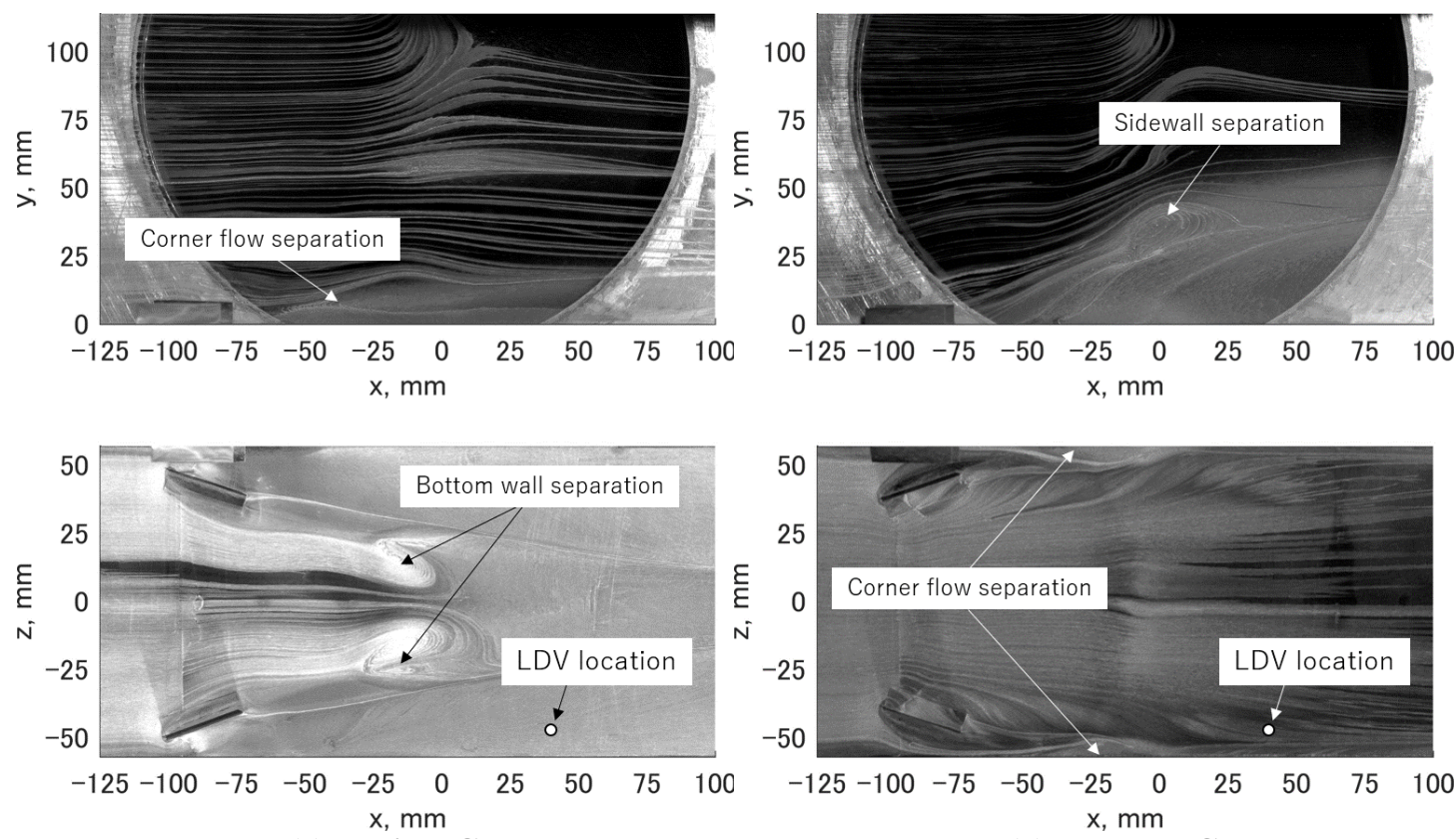

(a)Toe-in VGs

(b)Toe-out VGs

Figure 10 Oil flow pictures of VG base cases $\left(H_{v}=7.5 \mathrm{~mm}, D_{v t}=7.5 \mathrm{~mm}, X_{v s}=-100 \mathrm{~mm}\right)$.

(Top: sidewall at $z=+57 \mathrm{~mm}$, Bottom: bottom wall) 


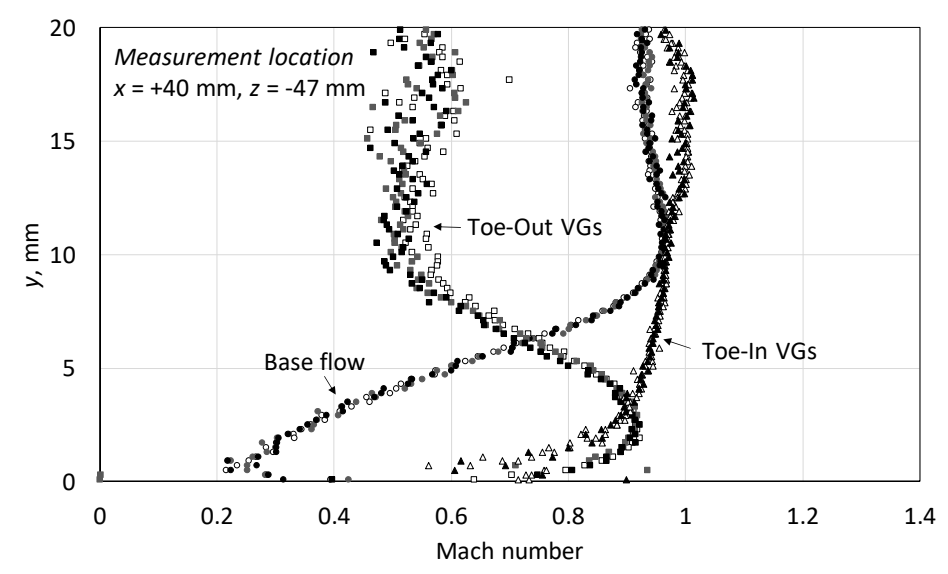

Figure 11 Local Mach number distribution downstream of the normal shock wave $(x=+40 \mathrm{~mm}, z=-$ $47 \mathrm{~mm})$.

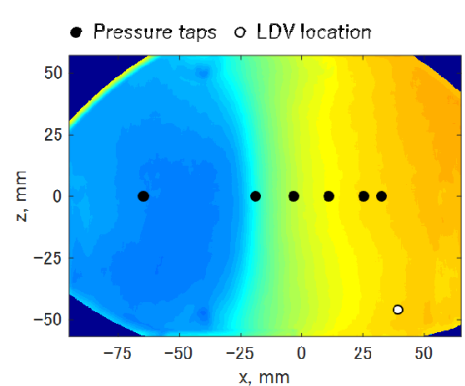

(a)Base flow

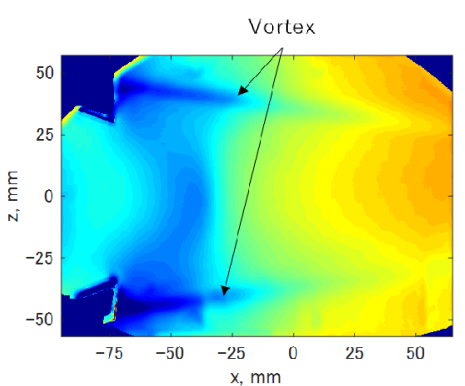

(b)Toe-in VGs

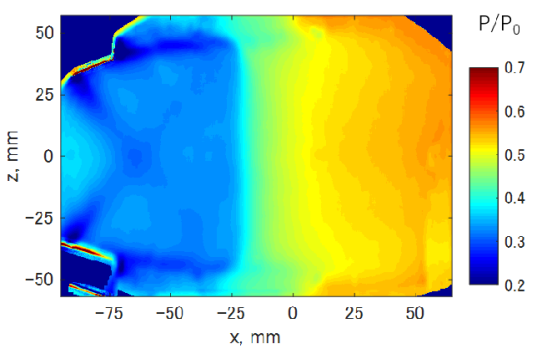

(c)Toe-out VGs

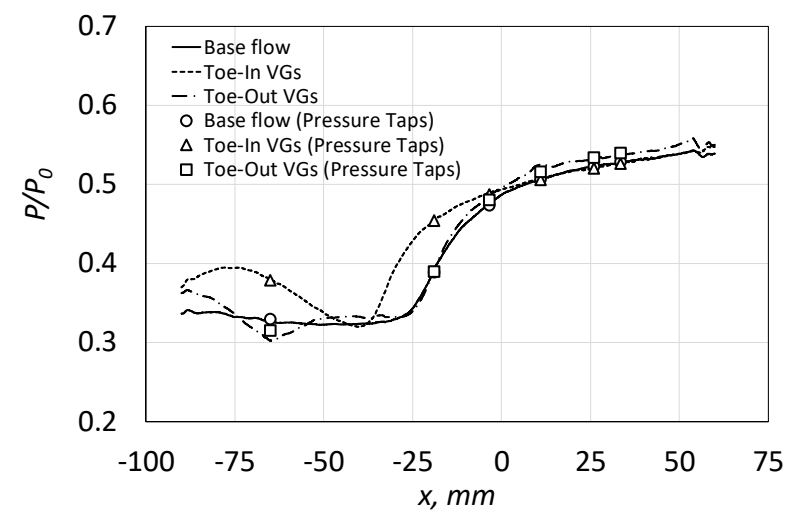

(d)Comparison between PSP and pressure tap data on the center line Figure 12 Pressure distribution on the bottom wall. 


\section{Influence of the VG size and the VG location}

As the size of the toe-in VGs decreases, the influence of the VGs on the flow field monotonically reduces as shown in Appendix 1. The size of the vortex and the bottom wall separation decreases gradually as the size of the VGs reduces.

On the other hand, the flow structure changes dramatically in the toe-out VGs cases when the size of the VGs decreases at the same location. Figure 13(a) shows the oil flow pictures in the smallest toe-out VGs case with a height of $2.5 \mathrm{~mm}$. The location is the same as that in Figs. $10-12$. The sidewall separation does not appear in this case. Instead, the size of the corner flow separation increases compared to the original size. The separation on the compression side of the vortex adheres to the corner flow separation.

To investigate the influence of the VGs location, the location of the smallest VGs is moved upstream from the previous location. Figure 13(b) shows the oil flow pictures for this case. The location of the VGs in the streamwise direction is $x=-200 \mathrm{~mm}$. The interval between the VG and the sidewall is the same as that in Fig. 13(a). From the oil flow on the bottom wall, it can be confirmed that the corner flow separation is reduced by the smallest VGs. An independent side wall separation can also be observed in the top figure of Fig. 13(b).

The influence of the interval between the VG and the sidewall is also investigated for the toe-out VGs. In these cases, the height of the VGs is $7.5 \mathrm{~mm}$. The location of the VGs in the streamwise direction is fixed at $x=-100 \mathrm{~mm}$. Figure 14(a) shows the oil flow for the largest interval case. The interval between the trailing edge of the VGs and the sidewall is $22.5 \mathrm{~mm}$. At this condition, an independent sidewall separation does not appear in the same way as in Fig. 13(a). Instead of that, the size of the corner flow separation increases from the original size. The separation on the compression side of the vortex adheres to the corner separation.

Figure 14(b) shows the oil flow pictures for the smallest interval case. The interval between the trailing edge of the VG and the sidewall is $0 \mathrm{~mm}$. For this condition, the pattern on the bottom wall doesn't differ greatly from that in Fig. 10(b). However, the side wall separation is largest in the toe-out VGs cases.
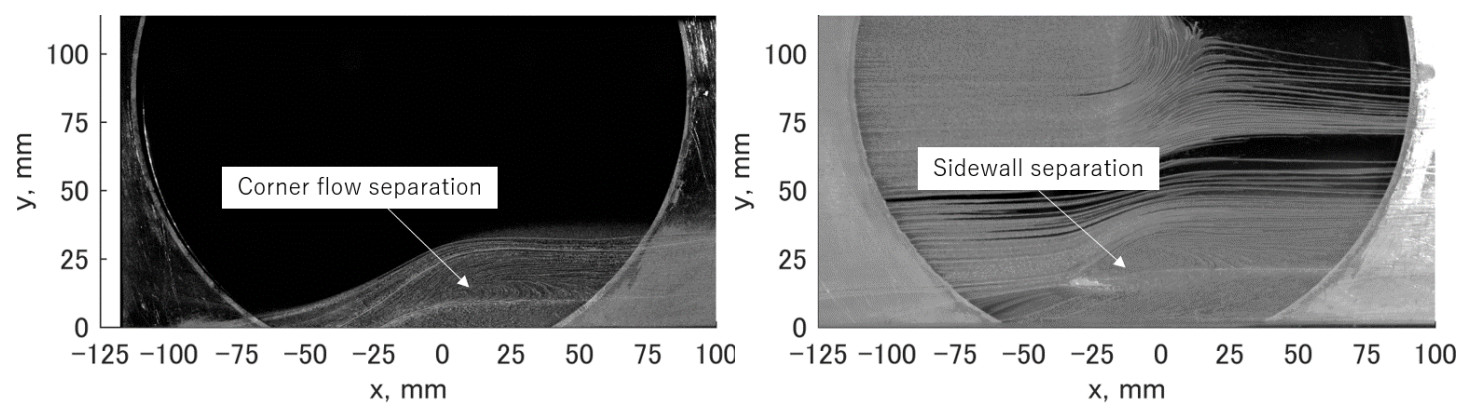

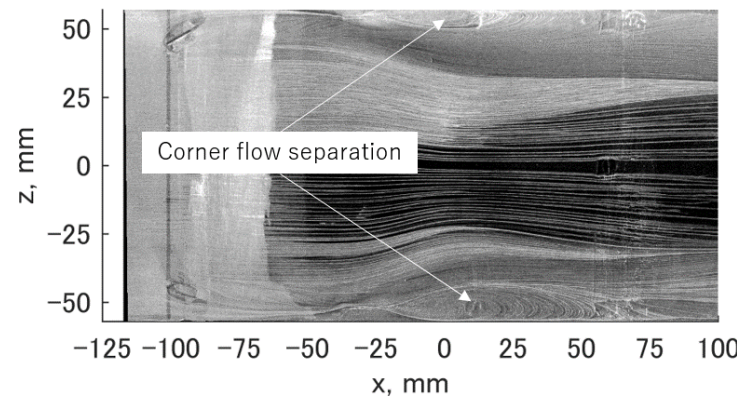

(a) $\Delta X_{v}=100 \mathrm{~mm}$

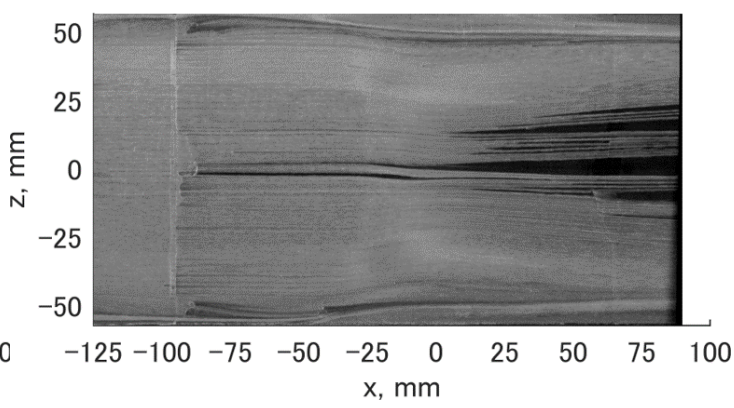

(b) $\Delta X_{v}=200 \mathrm{~mm}$

Figure 13 Oil flow pictures of smallest toe-out VGs $\left(H_{v}=2.5 \mathrm{~mm}, D_{v t}=7.5 \mathrm{~mm}\right)$. 

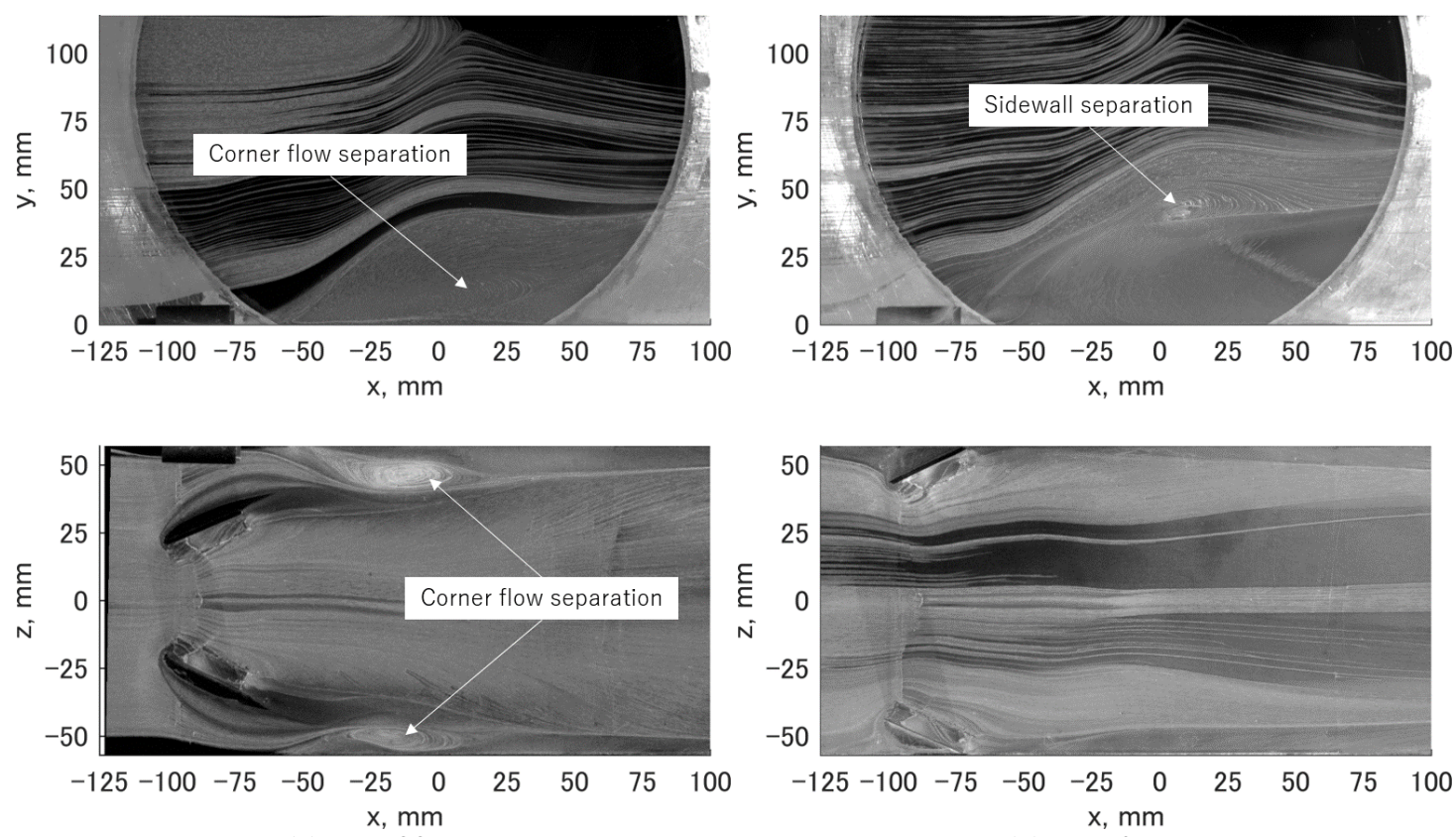

(a) $D_{v t}=22.5 \mathrm{~mm}$

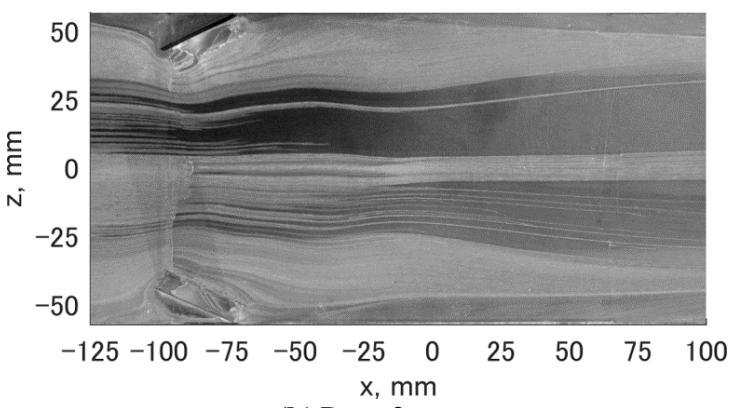

(b) $D_{v t}=0 \mathrm{~mm}$

Figure 14 Oil flow pictures of the toe-out VGs $\left(H_{v}=7.5 \mathrm{~mm}, \Delta X_{v s}=100 \mathrm{~mm}\right)$.

\section{Categorization of the separation mode}

We categorize the separation condition in the VGs cases based on the observation of the oil flow pictures. Figure 15 summarizes the categorization of the separation modes.

The modes in the toe-in VGs cases are simply grouped into the "influence mode" and the "no influence mode". When the size of the toe-in VGs decreases or the interval between the VG and the sidewall increases, the influence of the toe-in VGs on the corner flow decreases.

On the other hand, the separation modes in the toe-out VGs cases can be categorized into three modes. In the first mode, the sidewall separation appears independently. The size of the corner flow separation is reduced by the streamwise vortex. We name this first mode the "lift off mode" because the low momentum flow in the corner region is lifted off to high position on the sidewall. In the second mode, the corner flow separation increases from the base flow case. The sidewall separation does not appear in this mode. We name this second mode the "adhesion mode" because the low momentum flow on the compression side of the vortex adheres to the corner flow separation. The second mode appears when the VG size decreases and the interval between the VG and the sidewall increases from the condition in the lift off mode. The third mode is termed the "no influence mode". In this mode, the vortex does not interact with the corner flow separation. The bottom wall separation appears independently as shown in Fig. 7.

We categorize all VGs cases based on the definition of the separation modes. We summarize the results of the categorization in Appendix 1 to 3. 

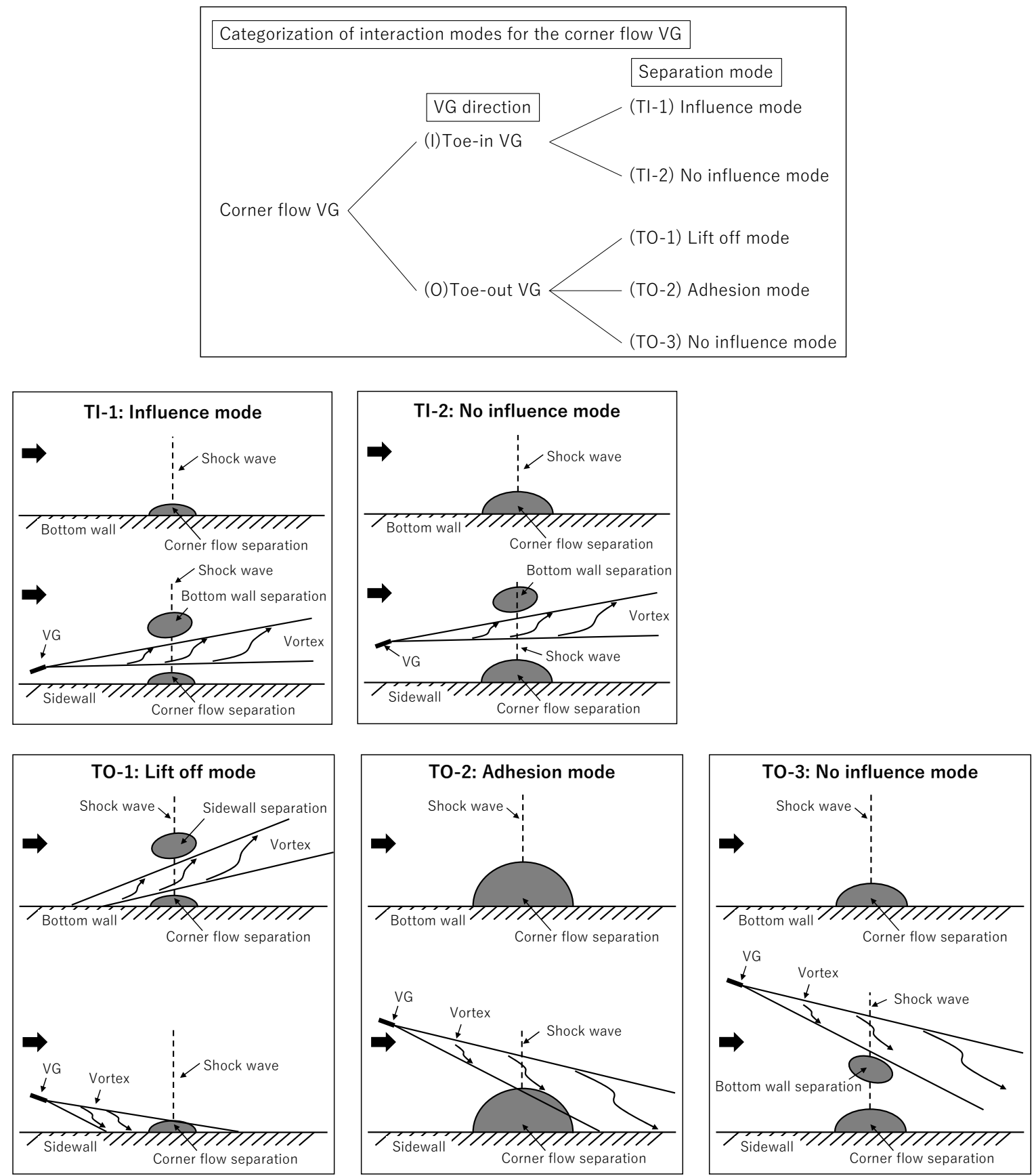

Figure 15 Categorization of the separation modes. 


\section{E. Quantitative evaluation of the VGs effect from oil flow pictures}

To evaluate the effect of the VGs quantitatively from the oil flow pictures, we introduce the evaluation, $W_{2 S}$ and $W_{2 B}$. We select streamlines at the widths $W_{1 S}$ and $W_{1 B}$ at $x=-60 \mathrm{~mm}$. The location of $x=-60 \mathrm{~mm}$ is chosen because it is downstream of the VGs trailing edge in all cases. The location of $y=10 \mathrm{~mm}$ and $z= \pm 47 \mathrm{~mm}$ are selected because the streamlines closer to the bottom wall and sidewall are not clear in the oil flow pictures. The location of $y$ $=57 \mathrm{~mm}$ is selected because it was the half width of the bottom wall. Both groups of streamlines had a local minimum width because of the corner flow separation. If the corner flow separation decreases, the local minimum width increases, and vice versa. Therefore, the local minimum width can be used as a measure of the effect of VGs on the corner flow separation. The evaluation $W_{2 B}$ is simply defined based on this concept because the streamlines on the bottom wall are symmetric. On the other hand, the streamlines on the sidewall have no restriction in the vertical direction. Hence, the evaluation $W_{2 S}$ is defined as the width between the streamline starting from $y=10 \mathrm{~mm}$ and the straight line of $y=57 \mathrm{~mm}$. When the sidewall separation or the bottom wall separation appears, $W_{2 S}$ and $W_{2 B}$ were defined as shown in Fig. 16(b). Thus, the values include the influence of the sidewall separation and the bottom wall separation.
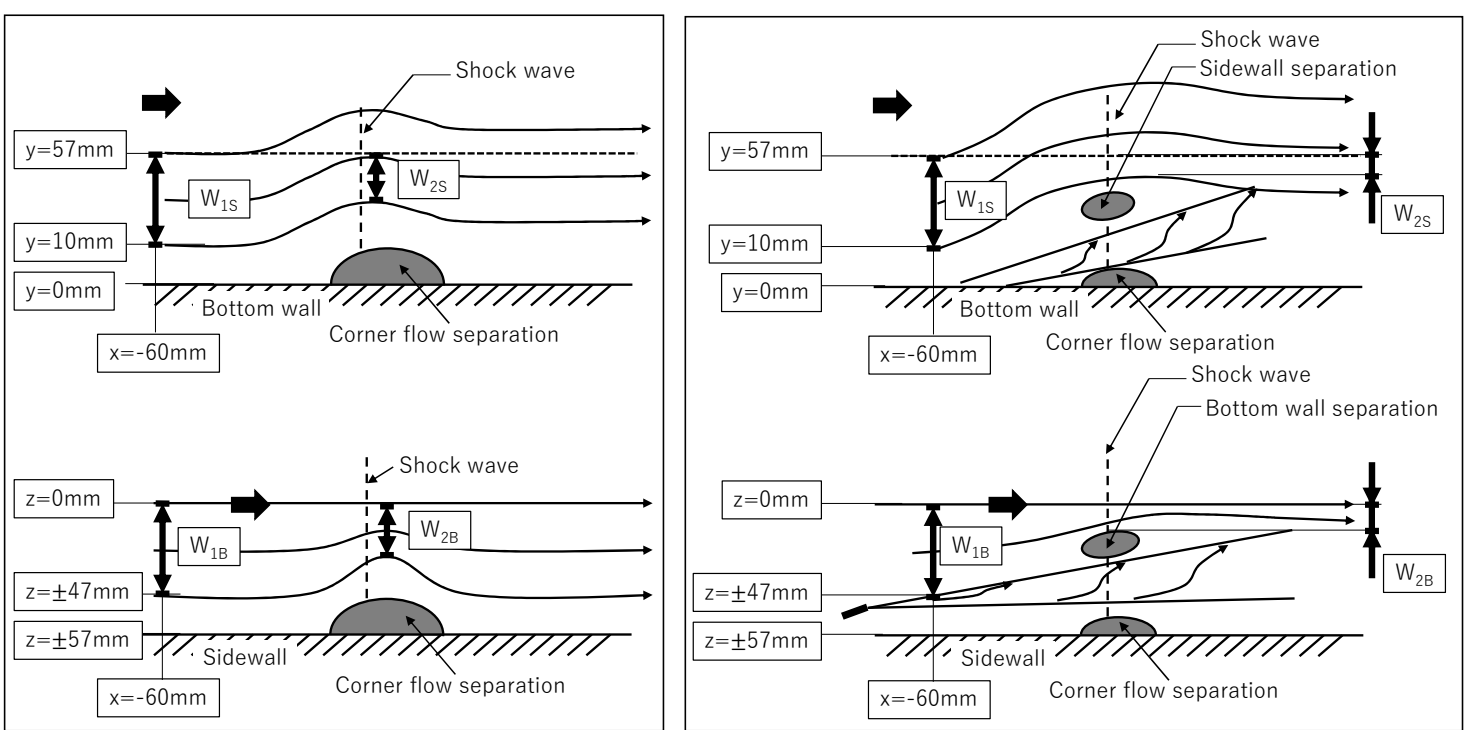

(a) No sidewall and bottom wall separation cases (b)Sidewall and bottom wall separation cases Figure 16 Definition of evaluation $W_{2 B}$ and $W_{2 S}$.

Figure 17 shows a comparison of the evaluation $W_{2 B} / W_{1 B}$ and $W_{2 S} / W_{1 S}$ between the base flow, the toe-in VGs $\left(H_{v}\right.$ $\left.=7.5 \mathrm{~mm}, \Delta X_{v s}=100 \mathrm{~mm}, D_{v l}=7.5 \mathrm{~mm}\right)$, and the toe-out VGs $\left(H_{v}=7.5 \mathrm{~mm}, \Delta X_{v s}=100 \mathrm{~mm}, D_{v t}=7.5 \mathrm{~mm}\right)$. In Fig. $17(\mathrm{a}), W_{2 B} / W_{1 B}$ in the toe-out VGs case is higher than other cases because the corner flow separation is reduced by the toe-out VGs. Because of the bottom separation, $W_{2 B} / W_{1 B}$ in the toe-in VGs is lower than other cases. On the other hand, in Fig. 17(b), the $W_{2 S} / W_{1 S}$ in the toe-out VGs case is lower than other cases because of the sidewall separation. The $W_{2 S} / W_{I S}$ in the toe-in VGs is higher than other cases because the corner flow separation is reduced by the VGs.

Figures 18 shows the influence of the VGs size on the evaluation in the toe-in VGs cases. Here, $W_{2 B}$ is normalized with the value in the base flow case, $W_{2 B_{-} B a s e}$. As mentioned above, the $W_{2 B}$ in the toe-in VGs cases is lower than that in the base flow case because of the bottom wall separation. As the VGs size increases, $W_{2 B} / W_{2 B} B a s e$ decreases monotonically.

Figures 19 shows the influence of the VGs size on the evaluation in the toe-out VGs cases. Here, $W_{2 B} / W_{2 B}$ Base is lowest at the lowest size in Fig. 19. At this point, the separation mode is the adhesion mode. When the VGs are higher than the boundary layer thickness, the separation mode is the lift off mode. In this mode, the value of the $W_{2 B} /$ $W_{2 B}$ Base $i$ is much greater than that in the adhesion mode. 
Figure 20 shows the influence of the distance between the VGs and the sidewall on the evaluation. The location in the uniform flow direction and the VGs size is fixed at $x=-100 \mathrm{~mm}$ and $H_{v}=7.5 \mathrm{~mm}$, respectively. As shown in Fig. 20(a), $W_{2 B} / W_{2 B_{-} \text {Base }}$ is high when the interval is small. At the largest interval, the separation mode is the adhesion mode. Because of the large corner flow separation, $W_{2 B} / W_{2 B}$ Base is lower than in the other cases. Figure 20(b) shows $W_{2 S} / W_{2 S}$ Base as a function of the interval. Because of the large sidewall separation, the value is lowest when the interval between the VG and the sidewall vanishes.

Figure 21 shows the influence of the VG location on the $W_{2 B} W_{2 B}$ Base for all cases except for the case with zero interval. The separation mode is the lift off mode when the VGs height is greater than the boundary layer thickness, and the parameter $\Delta X_{v s} / D_{v t}$ is higher than thirteen. In this mode, the value $W_{2 B} W_{2 B}$ Base decreases as the distance between the shock wave and the VGs $\Delta X_{v s}$ increases. It seems that the decrease in Fig. 21 is caused by the boundary layer development. When the height of the VGs is lower than the boundary layer thickness, $W_{2 B} / W_{2 B}$ Base is lower than in the other cases.

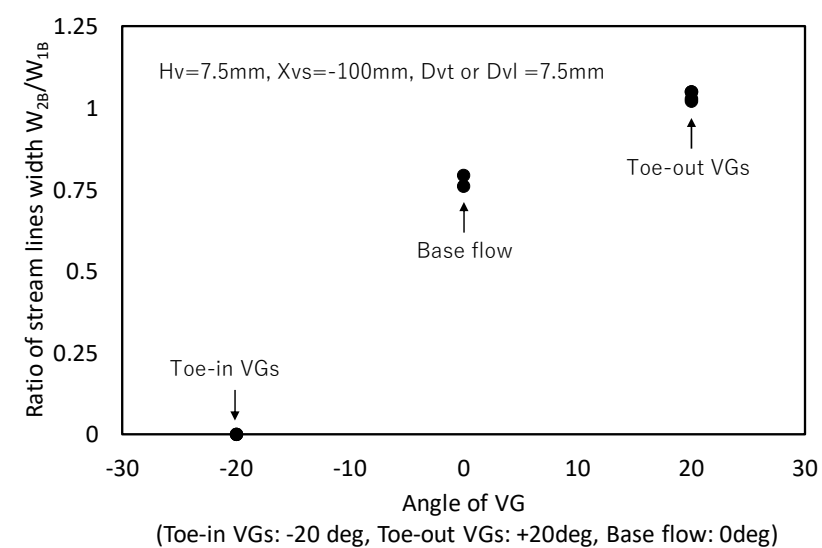

(a) Bottom wall

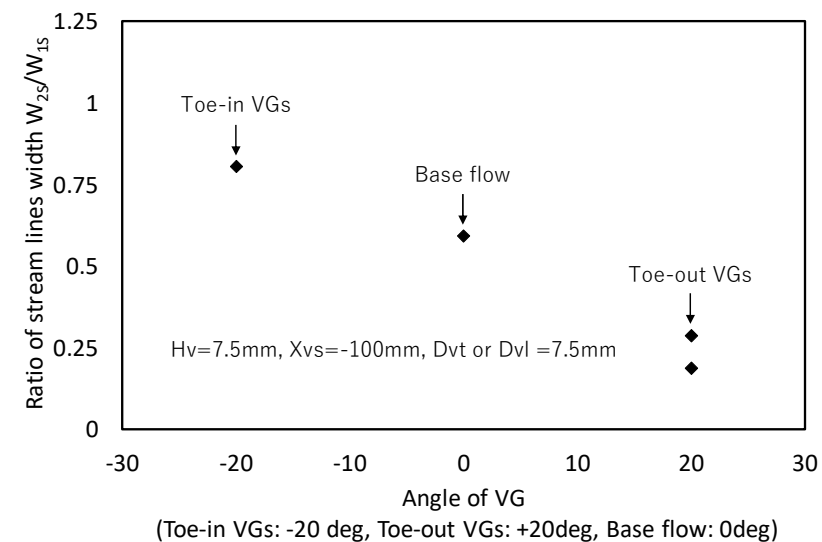

(b) Sidewall

Figure 17 Comparison of the evaluation $W_{2 B} / W_{1 B}$ and $W_{2 S} W_{1 S}$ between the base flow, the toe-in VGs $\left(H_{v}=7.5 \mathrm{~mm}, X_{v s}=-100 \mathrm{~mm}, D_{v l}=7.5 \mathrm{~mm}\right)$, and the toe-out VGs $\left(H_{v}=7.5 \mathrm{~mm}, X_{v s}=-100 \mathrm{~mm}, D_{v t}=\right.$ $7.5 \mathrm{~mm})$. 


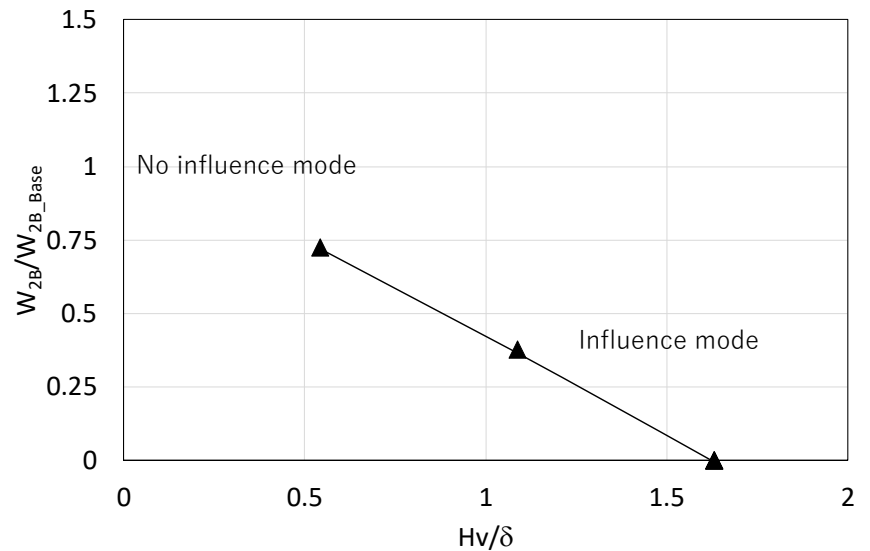

Figure 18 Influence of the height of the toe-in VGs $\left(X_{v s}=-100 \mathrm{~mm}, D_{v l}=7.5 \mathrm{~mm}\right)$ on the evaluation $W_{2 B} / W_{2 B_{-} \text {Base. }}$

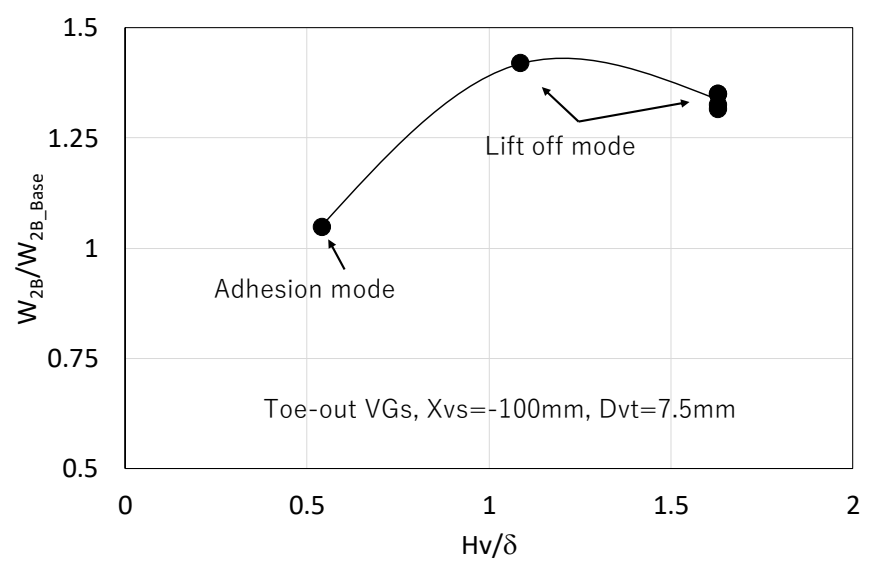

Figure 19 Influence of the height of the toe-out VGs $\left(X_{v s}=-100 \mathrm{~mm}, D_{v t}=7.5 \mathrm{~mm}\right)$ on the evaluation $W_{2 B} / W_{2 B}$ Base. 


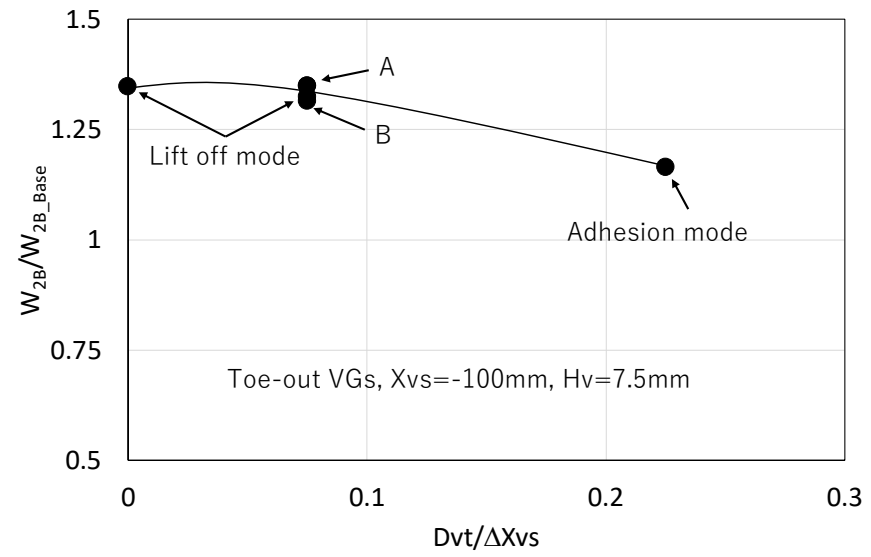

(a)Bottom wall

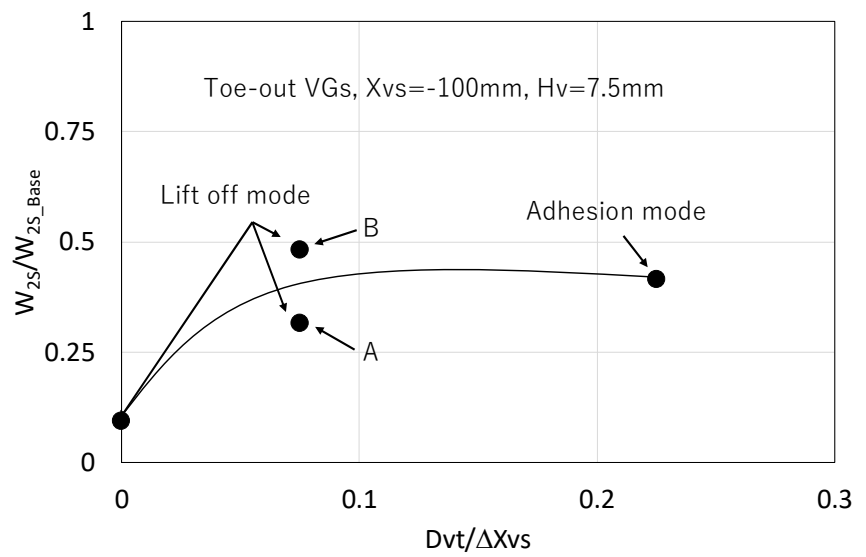

(b)Sidewall

Figure 20 Influence of the distance between the VG $\left(X_{v s}=-100 \mathrm{~mm}, H_{v}=7.5 \mathrm{~mm}\right)$ and the sidewall on the evaluation $W_{2 B} / W_{2 B_{-} \text {Base }}$ and $W_{2 S} / W_{2 S_{-} \text {Base. }}$

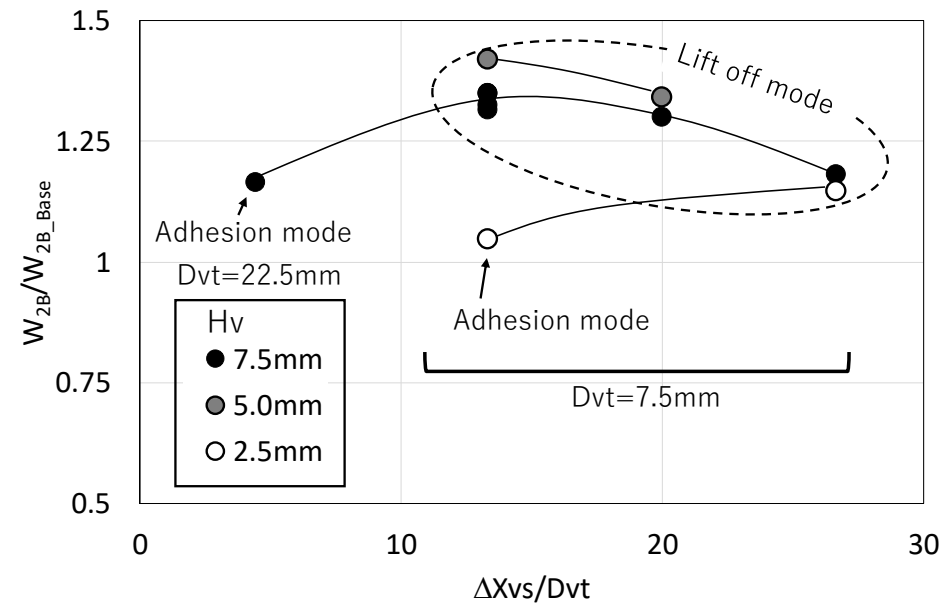

Figure 21 Relation between the evaluation $W_{2 B} / W_{2 B_{-} \text {Base }}$ and the VGs location parameter $\left(\Delta X_{v s} / D_{v t}\right)$. 
To summarize the influence of the size and location of the VGs on the evaluation $W_{2 B} W_{2 B_{-} B a s e}$ and the separation mode, we normalized the location parameter $\Delta X_{v s} / D_{v t}$ with the second power of the scale parameter $H_{v} / \delta$. Figure 22 shows $W_{2 B} W_{2 B_{3} \text { Base }}$ as a function of the parameters $\left(\Delta X_{v s} / D_{v t}\right)\left(H_{v} / \delta\right)^{2}$. This parameter successfully normalizes the influence of the VGs size. The boundary of the separation mode is at about $\left(\Delta X_{v s} / D_{v t}\right)\left(H_{v} / \delta\right)^{2}=14$. At this boundary, the $W_{2 B} W_{2 B_{B} \text { Base }}$ increased dramatically. After that, $W_{2 B} W_{2 B_{-} \text {Base }}$ decreases gradually because of the development of the boundary layer.

The normalization with the second power of the scale parameter is based on the idea shown in Fig. 23. In the flow fields, the trajectory and the vortex scale are influenced by the scale parameter $H_{v} / \delta$. In addition, it seems that the location of the sidewall separation is determined by the upper edge of the vortex. Figure 24 shows the trajectories of the vortex footprint. The height of the small $\mathrm{VG}$ and the large $\mathrm{VG}$ are $2.5 \mathrm{~mm}$ and $7.5 \mathrm{~mm}$, respectively. As shown in Fig. 24, the trajectory of the small VG is in good agreement with that of the large VG when they are both normalized with $H_{v} / \delta$. Hence, it seems that the idea in Fig. 23(a) is appropriate. Figure 23(a) shows this schematically. The normalization works quite well as shown in Fig. 22.

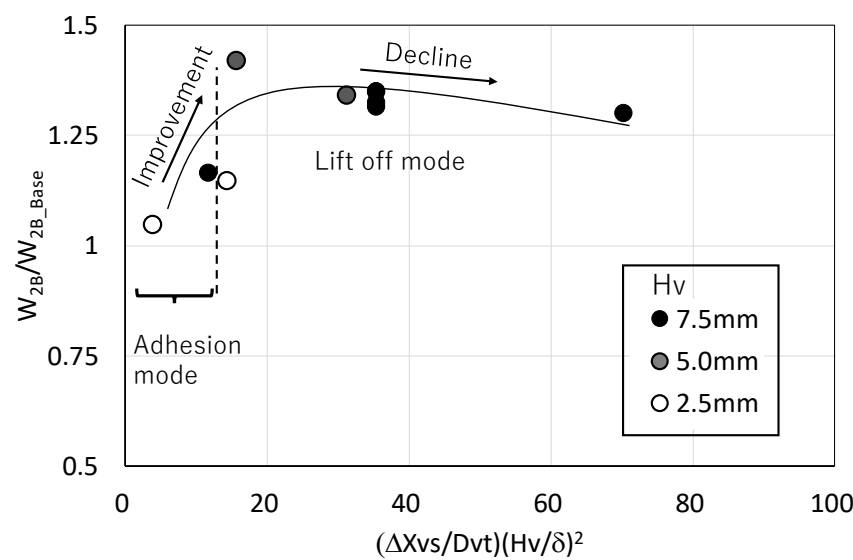

Figure 22 Relation between the evaluation $W_{2 B} W_{2 B_{-} \text {Base }}$ and the location parameter corrected by the VG scale $\left(\left(\Delta X_{v s} / D_{v t}\right)\left(H_{v} / \delta\right)^{2}\right)$.

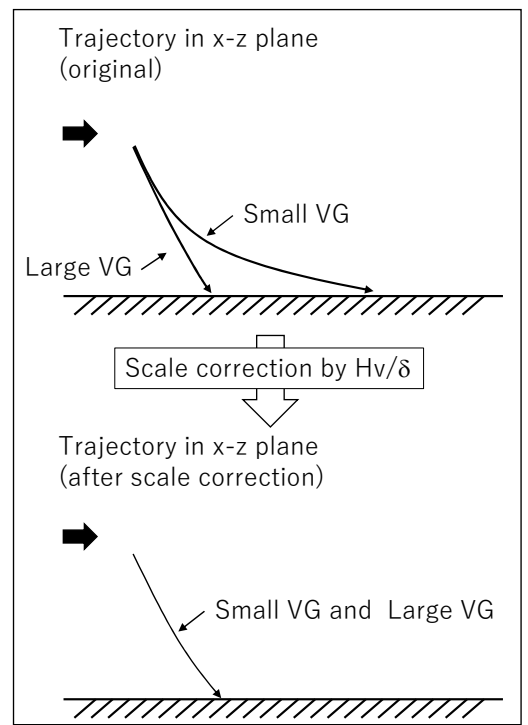

(a) Trajectory

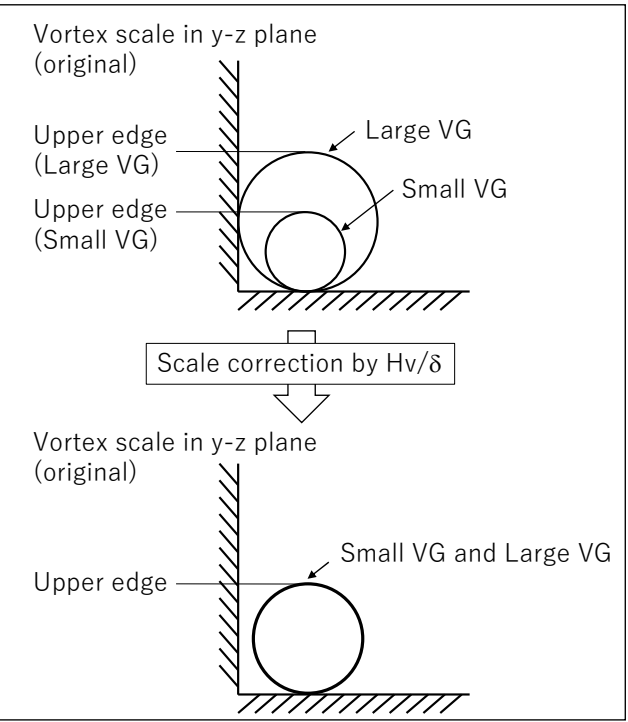

(b)Vortex scale

Figure 23 Conceptual figure of scale correction. 

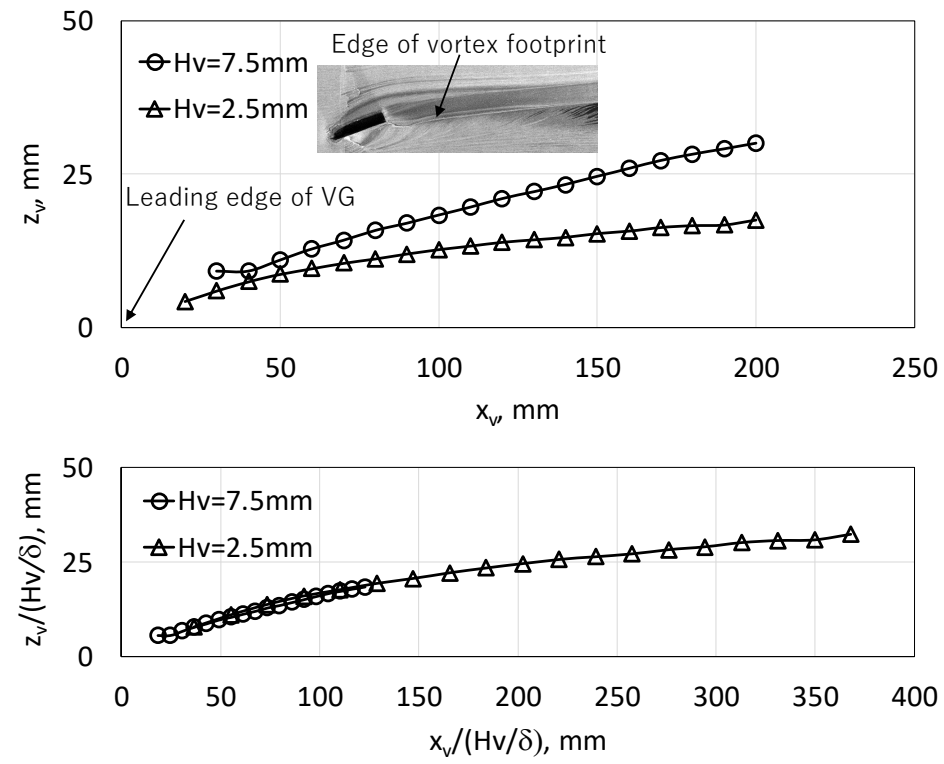

Figure 24 Trajectory of the vortex foot print.

\section{Conclusion}

Wind tunnel experiments were conducted to investigate the effect of vortex generators (VGs) on a corner flow separation caused by an interaction between a normal shock wave and the boundary layer in a Mach 1.4 flow. We defined VGs whose leading edge turns towards the corner as "toe-in VG", and VGs whose leading edge points in the opposite direction as "toe-out VG". The VGs were attached on the bottom wall of the test section. The influence of the VG direction, the size, and the location on the corner flow separation was investigated mainly based on oil flow pictures.

In all cases it was found that toe-in VGs reduced the corner flow separation. However, at the same time, a large separation appeared in the center region on the bottom wall. VGs whose height was about 1.5 times the boundary layer thickness reduced the corner flow separation when the interval between the VG and the sidewall was the same as the VGs height. The influence of the toe-in VGs monotonically decreased as the VGs size decreased.

The effect of the toe-out VGs was categorized in three "modes' namely, the "lift off mode", the "adhesion mode", and the "no influence mode". The lift off mode was effective at reducing the corner flow separation. However, this also caused a large sidewall separation. The effect of the VG decreased in the lift off mode with increasing distance from the shock wave. The area of the corner flow separation clearly increased in the adhesion mode.

We defined the ratio of the distance from the shock wave to the distance from the side wall as the location parameter. We also defined that the ratio of the VG height to the boundary layer thickness as the scale parameter. The effect of the toe-out VGs and the separation modes were successfully collapsed with the location parameter normalized by the second power of the scale parameter.

\section{Acknowledgement}

The authors would like to thank David Martin, Sam Flint and Anthony Luckett for their operation of the CUED blow-down wind tunnel. We also thank to Andrea Coschignano, Elise X. Xiang, Kshitij Sabnis, and Charlotte Coles for their cooperation in the experiment. Finally, we are grateful to Kazuyuki Nakakita and Daisuke Yorita for their advices in the PSP measurement.

\section{References}

${ }^{1}$ Bruce, P. J. K., Burton, B. M. F., Titchener, N. A., and Babinsky, H., "Corner Effect and Separation in Transonic Channel Flows," Journal of Fluid Mechanics, Vol. 679, 2011, pp. 247-262, doi:10.1017/jfm.2011.135.

${ }^{2}$ Pearcey, H. H., "Shock-Induced Separation and Its Prevention by Design and Boundary Layer Control," Boundary Layer and Flow Control, Edited by Lachmann, G. V., Vol. 2, Pergamon Press, pp. 1166-1344, 1961. 
${ }^{3}$ Ito, Y., Yamamoto, K., Kusunose, K., Koike, S., Nakakita, K., Murayama, M., and Tanaka, K., "Effect of Vortex Generators on Transonic Swept Wings," Journal of Aircraft, vol. 53, no. 6, 2016, pp. 1890-1904, DOI: 10.2514/1.C033737.

${ }^{4}$ Koike, S., Ito, Y., Kusunose, K, Nakajima, T., Sato, M., Kanda, H., Murayama, M., Nakakita, K., and Yamamoto, K., "Effect of Vortex Generators on Two-Dimensional Wings in Transonic Flows," JAXA-RR-14-002, 2014, ISSN 1349-1113. (in Japanese)

${ }^{5}$ CRM.65.airfoil sections, http://commonresearchmodel.larc.nasa.gov/crm-65-airfoilsections/[cited 24 June 2014].

${ }^{6}$ Hideaki Ogawa and Holger Babinsky, "Wind-Tunnel Setup for Investigations of Normal Shock Wave/Boundary Layer Interaction Control," AIAA Journal, Vol. 44, No. 11, 2006, pp. 2803-2805.

${ }^{7}$ Titchener, N., "An Experimental Investigation of Flow Control for Supersonic Inlets," Ph.D. Thesis, the University of Cambridge, 2013.

${ }^{8}$ Coschignano, A., Babinsky, H., Sheaf, C., and Platt, E., "Infuence of near-leading edge curvature on the performance of aeroengine intake lips at high-incidence," 34th AIAA Applied Aerodynamics Conference, AIAA AVIATION Forum, (AIAA 20163559), doi:10.2514/6.2016-3559, 2016.

${ }^{9}$ Liu, T. and Sullivan, J. P., Pressure and Temperature Sensitive Paints, Springer-Verlag Berlin Heidelberg, 2005.

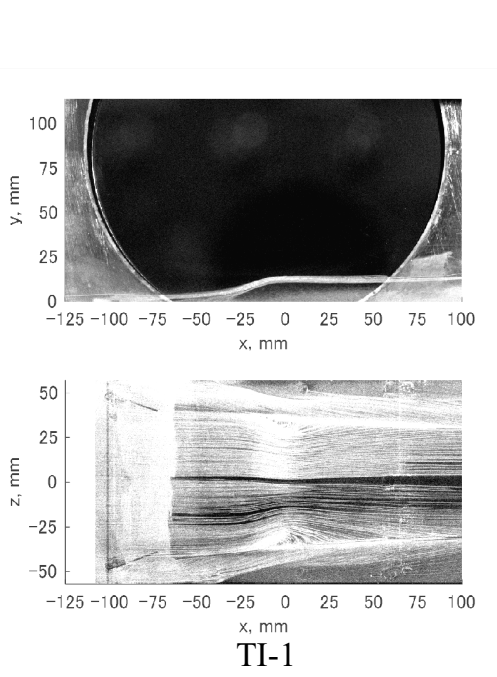

(a) $\mathrm{Hv}=2.5 \mathrm{~mm}$
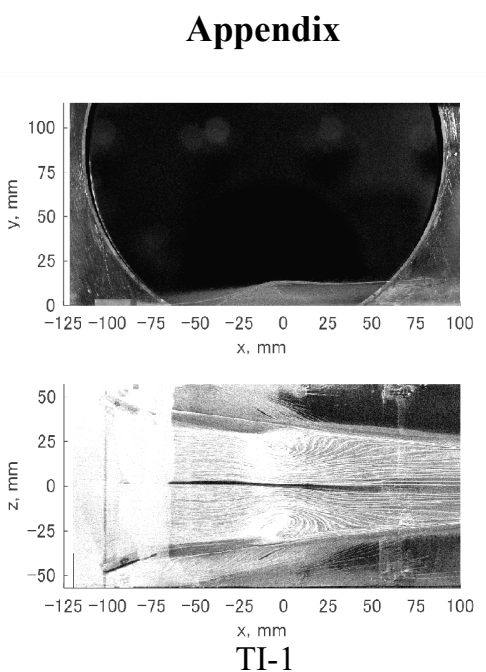

(b) $H v=5.0 \mathrm{~mm}$
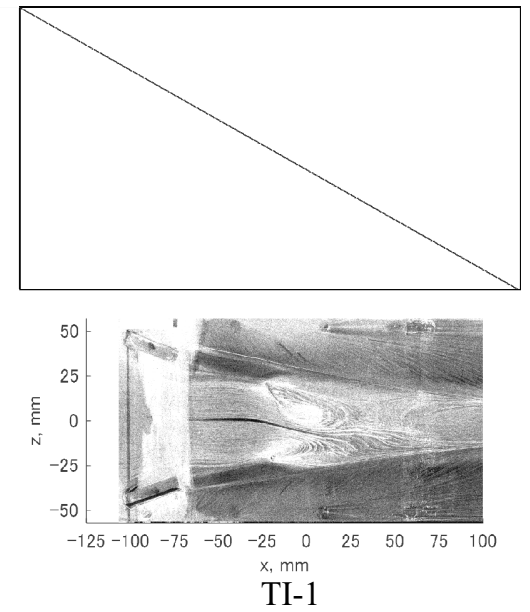

(c) $H v=7.5 \mathrm{~mm}$

Appendix 1 Oil flow pictures of toe-in VGs $\left(D_{v l}=7.5 \mathrm{~mm}, \Delta X_{v s}=100 \mathrm{~mm}\right)$
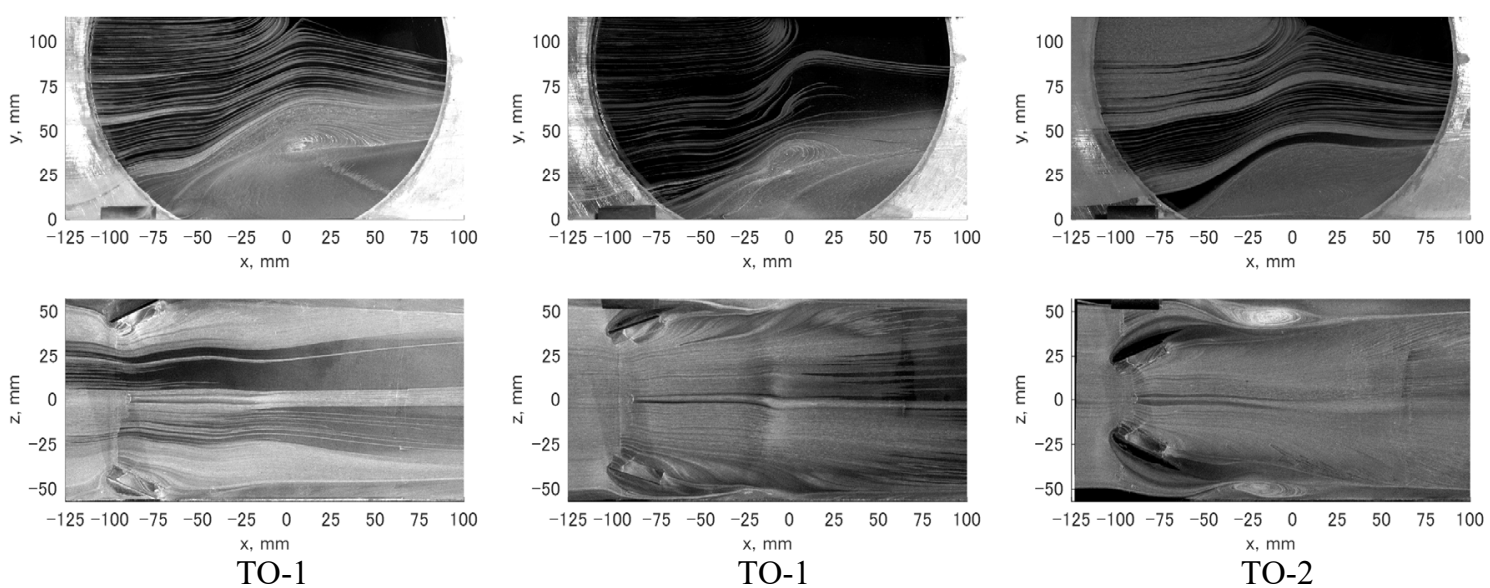

(a) $D_{v t}=0 \mathrm{~mm}$

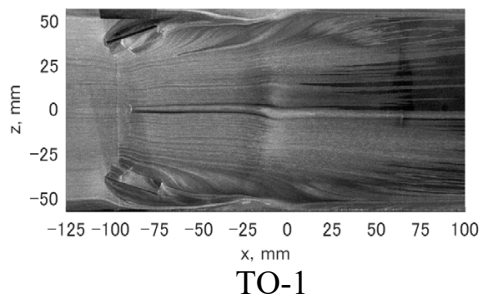

(b) $D_{v t}=7.5 \mathrm{~mm}$

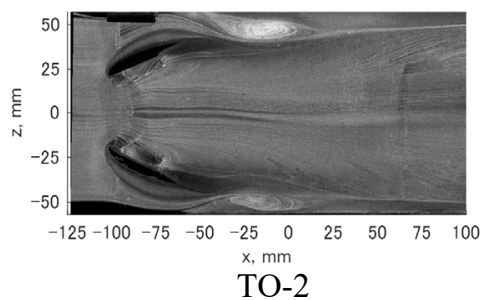

(c) $D_{v t}=22.5 \mathrm{~mm}$

Appendix 2 Oil flow pictures of toe-out VGs $\left(H_{v}=7.5 \mathrm{~mm}, \Delta X v s=100 \mathrm{~mm}\right)$ 

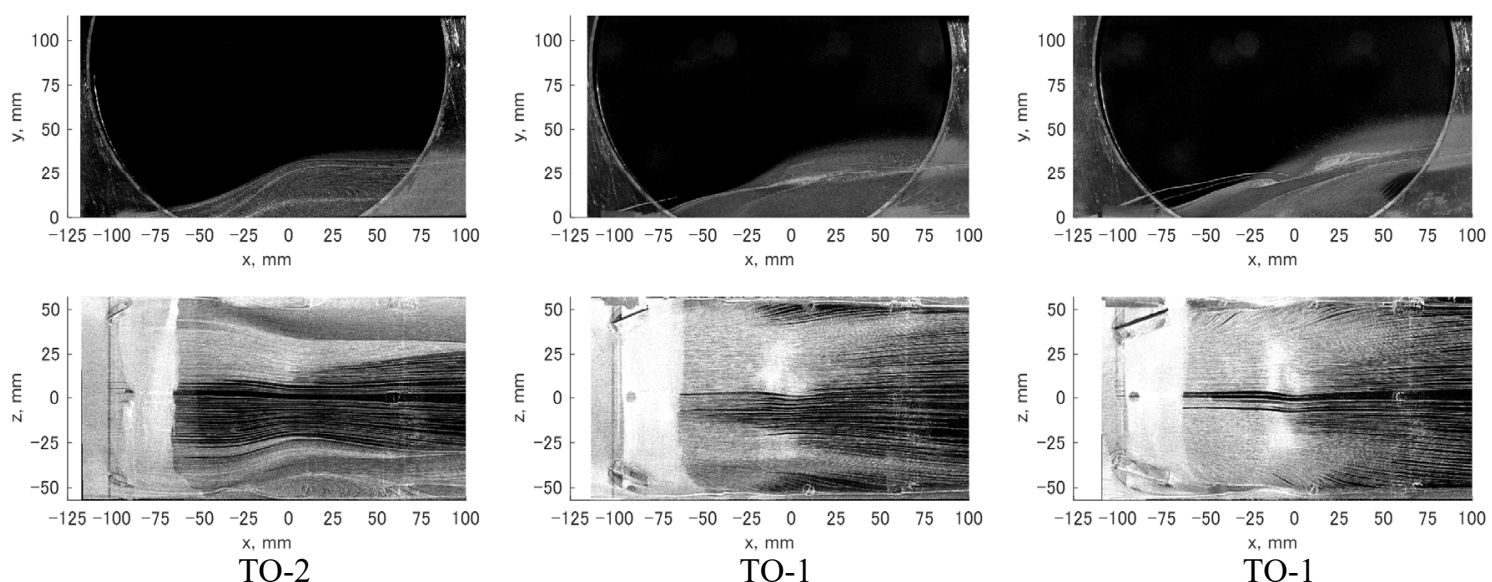

(a) $H v=2.5 \mathrm{~mm}, \Delta X_{v s}=100 \mathrm{~mm}$

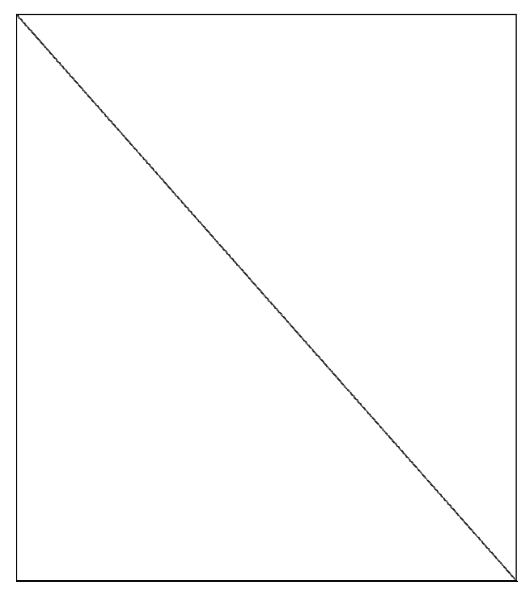

(b) $H v=5.0 \mathrm{~mm}, \Delta X_{v s}=100 \mathrm{~mm}$

(c) $H v=7.5 \mathrm{~mm}, \Delta X_{v s}=100 \mathrm{~mm}$
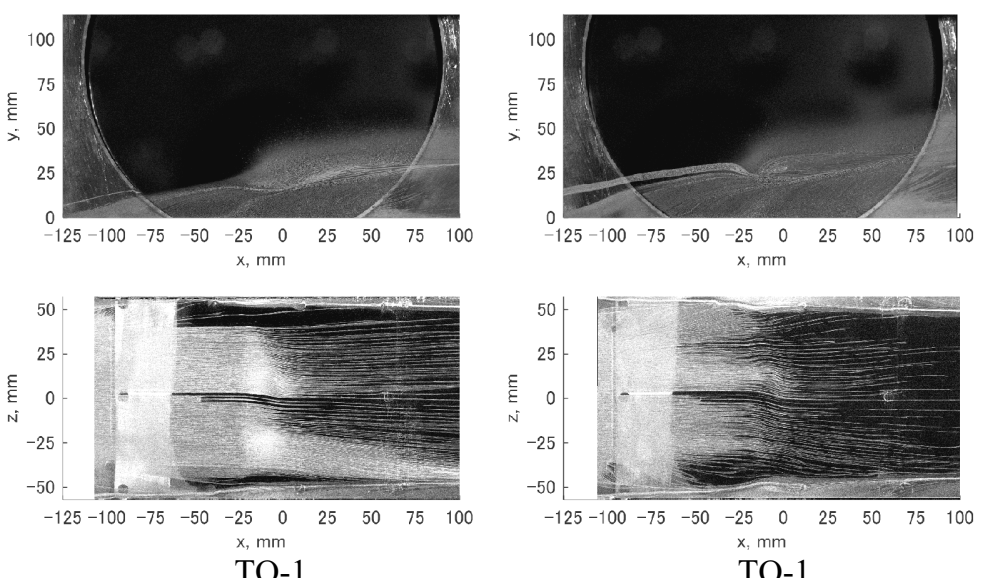

(d) $H v=2.5 \mathrm{~mm}, \Delta X_{v s}=150 \mathrm{~mm}$

(e) $H v=5.0 \mathrm{~mm}, \Delta X_{v s}=150 \mathrm{~mm}$

(f) $H v=7.5 \mathrm{~mm}, \Delta X_{v s}=150 \mathrm{~mm}$
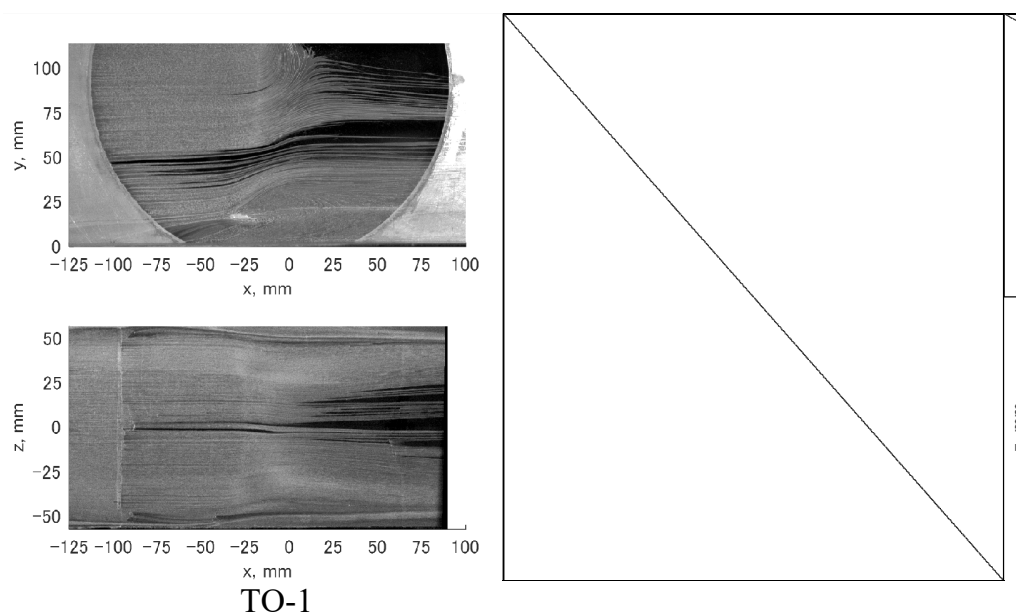

(g) $H v=2.5 \mathrm{~mm}, \Delta X_{v s}=100 \mathrm{~mm}$

(h) $H v=5.0 \mathrm{~mm}, \Delta X_{v s}=100 \mathrm{~mm}$ Appendix 3 Oil flow pictures of toe-out VGs $\left(D_{v t}=7.5 \mathrm{~mm}\right)$

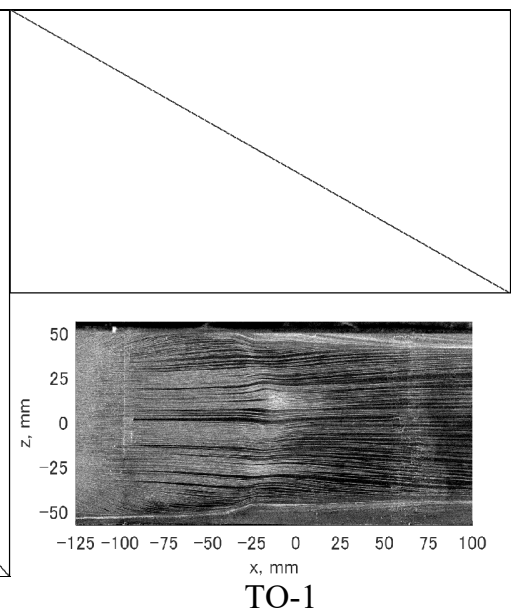

(i) $H v=7.5 \mathrm{~mm}, \Delta X_{v s}=100 \mathrm{~mm}$ 